\title{
NÉEL AND CROSS-TIE WALL ENERGIES FOR PLANAR MICROMAGNETIC CONFIGURATIONS
}

\author{
François Alouges ${ }^{1}$, Tristan Rivière ${ }^{2}$ and Sylvia Serfaty ${ }^{3}$
}

\begin{abstract}
We study a two-dimensional model for micromagnetics, which consists in an energy functional over $S^{2}$-valued vector fields. Bounded-energy configurations tend to be planar, except in small regions which can be described as vortices (Bloch lines in physics). As the characteristic "exchangelength" tends to 0, they converge to planar divergence-free unit norm vector fields which jump along line singularities. We derive lower bounds for the energy, which are explicit functions of the jumps of the limit. These lower bounds are proved to be optimal and are achieved by one-dimensional profiles, corresponding to Néel walls, if the jump is small enough (less than $\pi / 2$ in angle), and by two-dimensional profiles, corresponding to cross-tie walls, if the jump is bigger. Thus, it provides an example of a vector-valued phase-transition type problem with an explicit non-one-dimensional energy-minimizing transition layer. We also establish other lower bounds and compactness properties on different quantities which provide a good notion of convergence and cost of vortices.
\end{abstract}

Mathematics Subject Classification. 35J20, 35J60, 35Q60, 49S05, 49K20.

Received January 18, 2002.

\section{INTRODUCTION}

\subsection{General presentation}

This paper deals with the analysis of ferromagnetic materials. The general model for such materials is given by Brown [9] where it is considered that the sample occupies a region $\Omega \subset \mathbb{R}^{3}$ and there exists a distribution of magnetization $m(x) \in \mathbb{R}^{3}$ in $\Omega$ which is constrained to be of constant magnitude $M_{S}$, and to which is associated an energy of the form

$$
E(m)=A \int_{\Omega}|\nabla m|^{2}+K \int_{\Omega} \varphi(m)+\int_{\mathbb{R}^{3}}|H|^{2} .
$$

The first term is usually called the exchange energy and penalizes strong local variations of $m$.

The second term is called the anisotropy energy and accounts for favored directions for $m$, which are namely the wells of the smooth function $\varphi: S^{2} \rightarrow \mathbb{R}^{+}$. In real materials (see [21]), several anisotropies do exist, such

Keywords and phrases: Micromagnetics, thin films, cross-tie walls, Gamma-convergence.

1 Département de Mathématiques, Université de Paris-Sud, bâtiment 425, 91405 Orsay Cedex, France;

e-mail: Francois.Alouges@math.u-psud.fr

2 Department of Mathematics, ETH Zürich, 8092 Zürich, Switzerland; e-mail: riviere@math.ethz.ch

3 Courant Institute, 251 Mercer St., New York, NY 10012, USA, and supported by CNRS; e-mail: serfaty@cims.nyu.edu 
as uniaxial (one preferred direction), cubic (3 mutually orthogonal preferred directions), or planar (see [21], pp. 406 and 115).

Eventually, the last term is called the demagnetizing energy. The vector field $H$ is the stray field induced by the distribution in the whole space, given by (up to a physical factor)

$$
H=-\nabla \Delta^{-1} \operatorname{div}\left(m \mathbf{1}_{\Omega}\right),
$$

where $\mathbf{1}_{\Omega}$ denotes the characteristic function of $\Omega$.

It turns out that this term vanishes whenever $m$ is divergence-free and tangent to the boundary of $\Omega$. One can remark that $A$ must be proportional to the square of a length. This is the exchange length, typical lengthscale of order one variations of $m$.

There is a very large variety of configurations observed by physicists, which is partly explained by the competition between all these terms, the shape and size of the domain and various material constants and anisotropies. For instance, several possible limits are presented in detail in [15] with a particular focus on thin films.

There is also a fairly large mathematics literature on different variants of the energy (some include an applied magnetic field). First, Visintin [32] proved the existence of a solution of the system. The question of the smoothness of critical points was addressed in [10,19].

Many studies have focused on the "no-exchange" case where the exchange energy term is dropped ( $A$ set to zero): James and Kinderlehrer [23], DeSimone [12] (who justified the limit $A \rightarrow 0$ in the case of large bodies), Dacorogna and Fonseca [13]...

Very recently, in [16], DeSimone et al. have derived rigorously a 2D model as a thin-film limit of the full $3 \mathrm{D}$ model. They denote by $d$ the exchange-length, $l$ the side-length of the sample, and $t$ the thickness, and establish the Gamma-convergence in the regime $t \ll l$ and $d^{2} \log (l / t) / l t \ll 1$.

\subsection{The model}

The original model is 3D but we reduce to a $2 \mathrm{D}$ model by focusing on the case of an infinite cylindrical vertical body of section $\Omega$ and considering translation-invariant configurations. We also assume there is a forcing term which amounts to planar anisotropy, and that the lengthscale $\sqrt{A}$ is small compared to the size of the body. Mathematically, we are thus led to consider the functional

$$
E_{\varepsilon}(u)=\frac{1}{2}\left(\varepsilon \int_{\Omega}|\nabla u|^{2}+\frac{1}{\varepsilon} \int_{\mathbb{R}^{2}}|H|^{2}+\frac{1}{c_{\varepsilon}} \int_{\Omega}\left|u_{3}\right|^{2}\right),
$$

over $\Omega$ a smooth simply connected region of $\mathbb{R}^{2}$, where $u=\left(u_{1}, u_{2}, u_{3}\right) \in S^{2}$ is a unit vector field $\left(u=\frac{m}{M_{S}}\right)$, and $\varepsilon$ is a small lengthscale.

The demagnetizing field $H: \Omega \rightarrow \mathbb{R}^{3}$ is solution to

$$
\begin{cases}\operatorname{curl} H=0 & \text { in } \mathbb{R}^{2} \\ \operatorname{div}\left(H+u \mathbf{1}_{\Omega}\right)=0 & \text { in } \mathbb{R}^{2}\end{cases}
$$

and using the vertical translation invariance, one can easily observe that (1.4) implies that $H_{3}$ is uniform in space. Since $H$ belongs to $L^{2}\left(\mathbb{R}^{2}\right)$, we get $H_{3}=0$ and (1.4) rewrites into the two-dimensional model $H=\left(H_{1}, H_{2}\right)$,

$$
\begin{cases}\partial_{1} H_{2}-\partial_{2} H_{1}=0 & \text { in } \mathbb{R}^{2} \\ \partial_{1}\left(H_{1}+u_{1} \mathbf{1}_{\Omega}\right)+\partial_{2}\left(H_{2}+u_{2} \mathbf{1}_{\Omega}\right)=0 & \text { in } \mathbb{R}^{2}\end{cases}
$$

We want to study the asymptotics $\varepsilon \rightarrow 0$ (corresponding to a small lengthscale of variations of $u$ ), in the regime $c_{\varepsilon} \ll \varepsilon$, i.e. the planar anisotropy is stronger than the demagnetizing energy. For technical convenience, we will 
in fact assume

$$
c_{\varepsilon} \leq \varepsilon^{1+\delta} \quad \text { for some } 0<\delta<1
$$

In the limit $\varepsilon \rightarrow 0$, sequences of configurations of bounded energy $E_{\varepsilon}\left(u^{\varepsilon}\right) \leq C$ (such sequences do actually exist) converge to a limiting field $u$ that satisfies

$$
\left\{\begin{array}{l}
u \in S^{1} \text { i.e. } u=\left(u_{1}, u_{2}\right),|u|=1 \\
\operatorname{div} u=0 \text { in } \Omega \\
u \cdot n=0 \text { on } \partial \Omega
\end{array}\right.
$$

Indeed, observe that $u_{3} \rightarrow 0, H \rightarrow 0$ and then plug it into (1.5).

Writing $u=-\nabla^{\perp} g$ leads to the eikonal equation

$$
\begin{cases}|\nabla g|=1 & \text { in } \Omega \\ g=0 & \text { on } \partial \Omega .\end{cases}
$$

Such fields $u$ typically have line singularities on which the energy concentrates. This kind of phenomenon has been studied for related energies, see $[2-4,14,22,25]$ for the gradient theory of phase transitions or "Aviles-Giga" energy

$$
F_{\varepsilon}(u=\nabla \psi)=\int_{\Omega} \varepsilon|\nabla(\nabla \psi)|^{2}+\frac{1}{\varepsilon}\left(1-|\nabla \psi|^{2}\right)^{2},
$$

and $[30,31]$ for a $2 \mathrm{D}$ micromagnetic toy model. Beside the compactness proofs $\left(i . e . u^{\varepsilon} \rightarrow u\right.$ in $\left.\cap L^{p}\right)$ the essential problem is always to understand the structure and cost of jumps across the singular lines at the limit $\varepsilon \rightarrow 0$, a question which was more particularly addressed in $[2,22,30,31]$.

The model we study here may seem somewhat artificial, because of the vertical invariance and planar anisotropy assumptions. (As we mentioned, it corresponds to a correct limit of the full model in the rather unrealistic infinite-cylinder case.) However, it seems that it has a more general link with the full micromagnetics model, which still remains to be understood: this seems like an important open question. It is suggested by a formal argument following the type of argument of [15]: in relatively-thick thin-film regime, i.e. where the thickness is small but much larger than the exchange length $\sqrt{A}=\varepsilon$; without assuming any anisotropy, the two dimensional limiting energy should penalize the square of the $L^{2}$ norm of the $2 \mathrm{D}$ demagnetizing field $H$ (as in (1.3)) and should also penalize the vertical component of the magnetization, thus creating a planar forcing (as in (1.3) again).

What is clear is that the model we study here contains what seem like the essential ingredients which should appear in a thin-film micromagnetics model: it contains a nonlocal term which penalizes the divergence of the magnetization and a term penalizing the vertical component of the magnetization, thus it leads to the right limiting problem (1.7); moreover, it allows for the presence of vortices (see Sect. 1.4), which, although they carry only a vanishing fraction of the energy, are crucial for determining the energy of the global configuration.

Finally, as we will see in the rest of the paper, the results we obtain for this model present striking similarities with the observations (in particular those of cross-tie walls) made in soft ferromagnetic thin films. This is why we keep in this paper the terminology of the physics of ferromagnetic thin films. The good agreement between predictions of this model and experimental evidence support the conjecture that there should exist a rigorous link between this energy and the original one.

\subsection{The results of $[30,31]$}

Let us explain in further detail the mathematical model studied by Rivière and Serfaty in [30,31]. It consists in studying the same energy under the additional constraint that $u$ belongs to $S^{1}$ (i.e. $u_{3} \equiv 0$ and the anisotropy 
term drops out). In that case, any $u^{\varepsilon} \in H^{1}\left(\Omega, S^{1}\right)$ possesses a lifting $\varphi_{\varepsilon} \in H^{1}(\Omega, \mathbb{R})$ such that

$$
u^{\varepsilon}=\mathrm{e}^{i \varphi_{\varepsilon}} .
$$

The sequence $\left(\varphi_{\varepsilon}\right)$ is shown to be also compact and the authors exhibit a limiting energy which is written in terms of $\varphi$, the limit of $\varphi_{\varepsilon}$ :

$$
E_{0}(u, \varphi)=\int_{\Omega}\left|\operatorname{div}\left(\varphi u+u^{\perp}\right)\right|
$$

Here, $u^{\perp}=\left(-u_{2}, u_{1}\right)$, and $\operatorname{div}\left(\varphi u+u^{\perp}\right)$ is a bounded Radon measure supported on the singular set of $\varphi$ $\left(\int_{\Omega}\left|\operatorname{div}\left(\varphi u+u^{\perp}\right)\right|\right.$ is its total mass $)$. In the case $\varphi \in B V(\Omega, \mathbb{R}), E_{0}$ can be written in a more explicit way

$$
E_{0}(u, \varphi)=\int_{\Sigma_{\varphi}} 2|\sin X-X \cos X|
$$

where $\Sigma_{\varphi}$ is the one-dimensional jump set of $\varphi$ and $X=\frac{[\varphi]_{\Sigma_{\varphi}}}{2}$ is half the value of the jump of $\varphi$ across $\Sigma_{\varphi}$ (this formula is valid for $|X| \leq \pi$ and can be extended to all $X$, see [31]). This lower bound was proved to be optimal (at least for $|X| \leq \pi$ ) and achieved by one-dimensional profiles at the scale $\varepsilon$ called Néel walls in the physics literature.

Moreover, it is also proved in [30] that

$$
\min E_{0}(u, \varphi)=|\partial \Omega|, \quad \min _{u_{\varepsilon} \in H^{1}\left(\Omega, S^{1}\right)} E_{\varepsilon}\left(u^{\varepsilon}\right) \rightarrow|\partial \Omega|,
$$

and minimizers of $E_{\varepsilon}$ (under the $S^{1}$ constraint) converge to $u_{*}$ or $-u_{*}$ where

$$
u_{*}(x)=\nabla^{\perp} \operatorname{dist}(x, \partial \Omega),
$$

and $\nabla^{\perp}=\left(-\partial_{2}, \partial_{1}\right)($ see $[5])$.

Taking a minimizing sequence of the energy of [30] as a test-function in $E_{\varepsilon}$, we get as a first upper bound that $\min E_{\varepsilon} \leq|\partial \Omega|+o(1)$.

\subsection{Vortices}

Let us point out that the energy computed in [30] includes a cost (see (1.11)) for jumps of $2 \pi$ in $\varphi$ (while $u$ does not jump), and such jumps have to occur because of the topological constraint $u^{\varepsilon}(x) \in S^{1}$ and $u \cdot n=0$ on $\partial \Omega$. The model that we study here allows us to release this artificial constraint $u^{\varepsilon} \in S^{1}$, and thus lower energies can be achieved. We only have $u \in S^{1}$ at the limit, and this makes the situation quite different. Indeed, one can consider the planar projection of $u^{\varepsilon}$

$$
u_{\|}^{\varepsilon}=\left(u_{1}^{\varepsilon}, u_{2}^{\varepsilon}\right)
$$

It does not satisfy $u_{\|}^{\varepsilon} \in S^{1}$, and admits a local lifting $\varphi_{\varepsilon}$ such that

$$
u_{\|}^{\varepsilon}=\rho_{\varepsilon} \mathrm{e}^{i \varphi_{\varepsilon}}
$$

which cannot be defined globally since $\rho_{\varepsilon}=\left|u_{\|}^{\varepsilon}\right|$ can vanish at a point $x_{0}$ while $\varphi_{\varepsilon}$ can have a nonzero degree around $x_{0}$. This is precisely what is called a vortex, and such vortices are frequently seen in physical situations and called "Bloch lines". Mathematically, they can be compared to the vortices arising in the Ginzburg-Landau type models (see the book by Bethuel et al. [7]). Here, they carry only a vanishing fraction of the energy, and they allow to get rid of jumps of $2 \pi$ of the phase. 
In fact, the functionals

$$
\int_{\Omega} \varepsilon|\nabla u|^{2}+\frac{1}{c_{\varepsilon}}\left|u_{3}\right|^{2}
$$

with $u \in H^{1}\left(\Omega, S^{2}\right)$, corresponding to dropping $\int_{\mathbb{R}^{2}}|H|^{2}$ in $E_{\varepsilon}$, have been studied by Sandier [33], André and Shafrir [6], and more recently by Hang [20]. It leads to an analysis and to results that are very similar to [7].

Here, in contrast, because of the presence of the demagnetizing energy, which forces configurations to be almost divergence-free, $u$ not only has vortex (point) singularities, but also line singularities. Also we will see that the notion of vortex is weaker here, and, because $u$ has line singularities, the vortices cannot be defined from the knowledge of $u$ only, but rather as limits of those of $u^{\varepsilon}$, and will thus depend on the sequence $u^{\varepsilon} \rightarrow u$.

Let us now focus on the crucial example of $\Omega=B(0, R)$, the limiting divergence-free configuration $u_{*}=$ $\nabla^{\perp} \operatorname{dist}(x, \partial \Omega)=\mathrm{e}^{i \theta}$ in polar coordinates, can be approximated by a smooth $u_{\|}^{\varepsilon}=\rho_{\varepsilon} \mathrm{e}^{i \theta}$ with $\rho_{\varepsilon}(0)=0$, and such that the energy tends to 0 as $\varepsilon \rightarrow 0$. (In fact, the result of [24] is that the limiting energy can only be zero in the case of $\Omega$ being a disc and $u= \pm u_{*}$.)

On the other hand, trying to approximate $u_{*}$ by smooth $S^{1}$-valued $u^{\varepsilon}$ gives rise to a concentration of the energy along a radius of $B(0, R)$, along which $u^{\varepsilon}$ has to wind once around $S^{1}$. In that case (which is the case of [30]), the energy is bounded below by $2 \pi R$ (perimeter of the ball). This illustrates the main difference between both models and it turns out to be a major feature in the richness of limiting configurations, as well as new technical difficulties. For instance, the upper bound on the energy $E_{\varepsilon}\left(u^{\varepsilon}\right) \leq C$ is not sufficient to control the number of vortices at the limit, contrarily to cases such as [7]. However, the mathematical quantities and methods introduced in [30] turn out to be relevant for analyzing this new problem (the jump singularities and their cost).

\subsection{Main result}

The spirit is, exactly as in [30,31], that of Gamma-convergence: we look for relevant lower bounds for the energy and for corresponding upper bounds, thus deriving a limiting energy in the asymptotics $\varepsilon \rightarrow 0$.

Let us start from a family $u^{\varepsilon} \in H^{1}\left(\Omega, S^{2}\right)$ such that $E_{\varepsilon}\left(u^{\varepsilon}\right) \leq C$. The $L^{p}$ compactness of $u^{\varepsilon}$ follows easily from previous studies on similar problems (for example the proof of [25], see Lem. 2.1), and $u^{\varepsilon}$ converges strongly to a limiting $S^{1}$-valued $u$ which satisfies (1.7), and which generically has line-singularities.

If $u$ is assumed to be in $B V(\Omega)$, then we can get a lower bound for the energy in terms of the jumps of $u$, which is of the type of (1.11), but a different formula.

The structure theorem of $B V$ allows to define a rectifiable one-dimensional jump set of $u, \Sigma_{u}$, and traces $u_{+}, u_{-}$on both sides of $\Sigma_{u}$. Then we define

$$
X=\frac{1}{2} \min \left|\left(\widehat{u_{ \pm} ; u_{\mp}}\right)\right| \in\left[0, \frac{\pi}{2}\right]
$$

i.e. the geometric half-angle between $u_{+}$and $u_{-}$.

Theorem 1. Let $\varepsilon_{n} \rightarrow 0$ and $u^{\varepsilon_{n}}$ be sequences of maps from $\Omega$ into $S^{2}$ satisfying $E^{\varepsilon_{n}}\left(u^{\varepsilon_{n}}\right) \leq C$. Then, up to extraction, $u^{\varepsilon_{n}}$ converges in $\cap_{p<\infty} L^{p}(\Omega)$ to some limit $u$ which satisfies (1.7). If, in addition, $u \in B V\left(\Omega, S^{1}\right)$, then

$$
\liminf _{n \rightarrow \infty} E_{\varepsilon_{n}}\left(u^{\varepsilon_{n}}\right) \geq \int_{\Sigma_{u}} A(X) \mathrm{d} \mathcal{H}^{1}
$$

where

$$
\begin{aligned}
& A(X)=2|\sin X-X \cos X| \quad \text { for } X \in\left[0, \frac{\pi}{4}\right] \\
& A(X)=2\left|\left(X-\frac{\pi}{2}\right) \cos X-\sin X+\sqrt{2}\right| \quad \text { for } X \in\left[\frac{\pi}{4}, \frac{\pi}{2}\right] .
\end{aligned}
$$

Here, $\mathcal{H}^{1}$ denoted the one-dimensional Hausdorff measure. 
One notices that for jumps less than $\frac{\pi}{4}(1.16)$, one recovers (1.11) and thus the (purely planar) Néel walls found in [30] are still optimal. On the contrary, for jumps bigger than $\frac{\pi}{2}$, they are no longer optimal (also in contrast with the case of the Aviles-Giga problem), the lower bound (1.17) is strictly smaller than that of (1.11), due to the extra degree of freedom $u^{\varepsilon} \in S^{2}$ and the possibility of vortices. The formula (1.17) is obtained by analytical means but turns out to be also optimal, which is one of our main results. Thus $\int_{\Sigma_{u}} A(X)$ can be seen as the limiting energy for $E_{\varepsilon}$.

Theorem 2. For all $X \in\left[\frac{\pi}{4}, \frac{\pi}{2}\right]$, there exists a "cross-tie wall" configuration u over $\mathbb{R}^{2}=\{(x, y)\}$ such that

$$
\begin{aligned}
\lim _{y \rightarrow+\infty} u(x, y) & =u_{+}=\mathrm{e}^{i\left(\frac{\pi}{2}-X\right)} \\
\lim _{y \rightarrow-\infty} u(x, y) & =u_{-}=\mathrm{e}^{i\left(\frac{\pi}{2}+X\right)},
\end{aligned}
$$

which is periodic in $x$, and whose energy per unit-length of $x$ is (after rescaling at any scale going to 0 )

$$
A(X)+o(1)=2\left|\left(X-\frac{\pi}{2}\right) \cos X-\sin X+\sqrt{2}\right|+o(1)
$$

The optimal configurations are this time two-dimensional configurations (i.e. $u$ depends on both $x$ and $y$ ) and have an unbounded number of vortices. They are observed in thin film experiments (see $[21,28,29]$ ) and are called in physics "cross-tie walls".

In the particular case $X=\frac{\pi}{2}$ the pattern is that of Figure 1.

The configuration is periodic and each cell contains one degree +1 and one degree -1 vortex (indicated by circles). This particular configuration is strikingly similar to the numerical simulations and experimental results presented in [28]. This picture can be generalized to all jumps $X \in\left[\frac{\pi}{4}, \frac{\pi}{2}\right]$, see Section 4.2.

One may then use these $2 \mathrm{D}$ cross-tie profiles to construct test-configurations which achieve equality in (1.15). Jump-lines of the cross-tie walls have to be regularized as 1D Néel walls (the jumps are $\leq \frac{\pi}{2}$ ). Since the energy per unit length of the cross-tie pattern is scale-invariant, one can blow them down and, given a $u$ with given jump-lines and jumps, paste them along the jump-lines at any scale which tends to 0 as $\varepsilon \rightarrow 0$ and which is $\gg \varepsilon$. This will regularize $u$ into a family $u^{\varepsilon} \rightarrow u$ with equality in (1.15).

The method to obtain the lower bound is inspired by the following "trick" of [30]

$$
E_{\varepsilon}\left(u^{\varepsilon}\right) \geq \frac{1}{2} \int_{\Omega} \varepsilon\left|\nabla u^{\varepsilon}\right|^{2}+\frac{1}{\varepsilon}\left|H_{\varepsilon}\right|^{2} \geq \int_{\Omega}\left|\nabla \varphi_{\varepsilon} \cdot H_{\varepsilon}\right| .
$$

The problem here is that the phase $\varphi_{\varepsilon}$ of $u^{\varepsilon}$ is not univalued, so we use the fact that

$$
E_{\varepsilon}\left(u^{\varepsilon}\right) \geq \int_{\Omega}\left|f\left(\varphi_{\varepsilon}\right) \nabla \varphi_{\varepsilon} \cdot H_{\varepsilon}\right|=\int_{\Omega}\left|\nabla F\left(\varphi_{\varepsilon}\right) \cdot H_{\varepsilon}\right|
$$

for all $|f| \leq 1$ and $F^{\prime}=f$. We then find such functions $F$ such that $F\left(\varphi_{\varepsilon}\right)$ depends only on $\varphi_{\varepsilon}$ modulo $2 \pi$, hence only on $u^{\varepsilon}$. As in [30], one can write $F\left(u_{\varepsilon}\right) \cdot H_{\varepsilon}$ as a divergence and pass to the limit (see Sect. 2).

The problem we have studied is a singularly perturbed (nonlocal) variational problem. It can be viewed as some kind of a phase-transition model (it is also a Landau theory) like (1.9) or its real-valued version (see $[27,34]$ ), where the transition layers are the singular lines across which our $u$ jumps. In real-valued phase transition models, the transitions are known to follow a one-dimensional profile. It is also the case in the problem of [30], and is what is expected for the Aviles-Giga energy (1.9), for which one knows that the onedimensional profile remains optimal. For vector-valued order parameters, it is known that energy-minimizing transition layers can sometimes be multidimensional rather than one-dimensional (e.g. Jin and Kohn [22], Conti et al. [11]) but here the achievement is that we are able to identify precisely the structure of an optimal non-one-dimensional transition layer structure. 


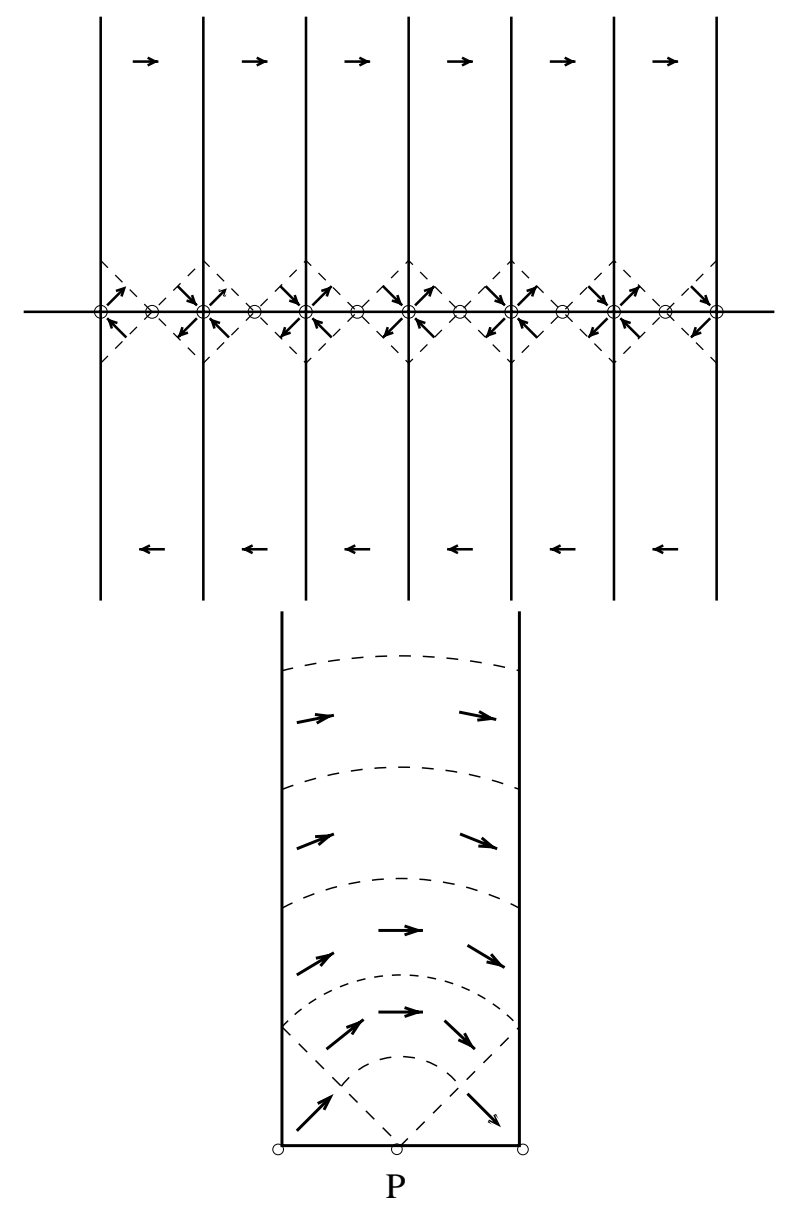

FiguRE 1. The optimal cross-tie wall.

\subsection{Lower bounds involving the vortices}

This second approach gives a result which is not always optimal but does not require any $B V$ assumption on $u$ and allows to take into account the vortices. The lower bound of Theorem 1 depends only on the limiting $u$. However, the result we are going to state shows that two sequences $u^{\varepsilon}$ converging to the same $u$ do not necessarily have the same limiting energy. Their vortex-structure, which is not given by the knowledge of $u$ (because $u$ has line singularities) has to be taken into account. One must thus add the knowledge of the vortices of the sequence $u^{\varepsilon} \rightarrow u$ and find a suitable notion of convergence of those vortices.

Let us start from a family $u^{\varepsilon} \in H^{1}\left(\Omega, S^{2}\right)$ such that $E_{\varepsilon}\left(u^{\varepsilon}\right) \leq C$, converging to $u$ in $L^{p}$.

By a certain transformation (see Lem. 2.2), one may reduce to configurations $u^{\varepsilon} \in H^{1}\left(\Omega \backslash\left\{a_{1}^{\varepsilon}, \cdots, a_{n(\varepsilon)}^{\varepsilon}\right\}, S^{1}\right)$, $S^{1}$-valued except at a finite number of singular points. First, from the equation (1.5) defining $H$, there exists $g_{\varepsilon}$ such that

$$
u^{\varepsilon} \mathbf{1}_{\Omega}+H_{\varepsilon}=-\nabla^{\perp} g_{\varepsilon}
$$

Notice that $g_{\varepsilon} \rightarrow g$ defined in (1.8). Away from its singular points, $u^{\varepsilon}$ admits locally an $H^{1}$-lifting $\varphi_{\varepsilon} \in \mathbb{R} i . e$. $u^{\varepsilon}=\mathrm{e}^{i \varphi_{\varepsilon}}$, by the result of [8]. The vector field $V_{\varepsilon}=\nabla \varphi_{\varepsilon}$ is curl-free in $\Omega \backslash \cup_{i}\left\{a_{i}\right\}$. Let also d $\mathrm{d}_{i}^{\varepsilon}$ be the degree associated to $a_{i}^{\varepsilon}$, i.e. the winding number of $\varphi_{\varepsilon}$ around $a_{i}$. 
The approach to the lower bound is again to start with (1.18). The idea of [30] was to write

$$
\nabla \varphi_{\varepsilon} \cdot H_{\varepsilon}=\nabla \varphi_{\varepsilon} \cdot\left(H_{\varepsilon}+u^{\varepsilon}\right)-\nabla \varphi_{\varepsilon} \cdot u^{\varepsilon}=-\nabla \varphi_{\varepsilon} \cdot \nabla^{\perp} g_{\varepsilon}-\operatorname{curl} u^{\varepsilon} .
$$

Now in the presence of vortices, we have

$$
-\nabla \varphi_{\varepsilon} \cdot \nabla^{\perp} g_{\varepsilon}-\operatorname{curl} u^{\varepsilon}=\operatorname{curl}\left(g_{\varepsilon} V_{\varepsilon}-u^{\varepsilon}\right)-2 \pi \sum_{i} \mathrm{~d}_{i}^{\varepsilon} g_{\varepsilon}\left(a_{i}^{\varepsilon}\right) \delta_{a_{i}^{\varepsilon}}
$$

where $\delta_{a_{i}}$ is the Dirac mass at the point $a_{i}$. If the vortices $\left(a_{i}, d_{i}\right)$ are fixed for example, one may write

$$
\begin{aligned}
\liminf _{\varepsilon \rightarrow 0} E_{\varepsilon}\left(u^{\varepsilon}\right) \geq \liminf _{\varepsilon \rightarrow 0} \int_{\Omega}\left|\nabla \varphi_{\varepsilon} \cdot H_{\varepsilon}\right| & \geq \int_{\Omega}\left|\operatorname{curl}\left(g_{\varepsilon} V_{\varepsilon}-u^{\varepsilon}\right)-2 \pi \sum_{i} \mathrm{~d}_{i} g_{\varepsilon}\left(a_{i}\right) \delta_{a_{i}}\right| \\
& =\int_{\Omega \backslash \cup_{i}\left\{a_{i}\right\}}\left|\operatorname{curl}\left(g_{\varepsilon} V_{\varepsilon}-u^{\varepsilon}\right)\right| .
\end{aligned}
$$

In the case where $g$ has a constant sign in $\Omega$, then $u \cdot \tau$ does not change sign on $\partial \Omega$ and formally passing to the limit $\varepsilon \rightarrow 0$,

$$
\liminf _{\varepsilon \rightarrow 0} E_{\varepsilon}\left(u^{\varepsilon}\right) \geq \liminf _{\varepsilon \rightarrow 0}\left|\int_{\Omega} \operatorname{curl}\left(g_{\varepsilon} V_{\varepsilon}-u^{\varepsilon}\right)-2 \pi \sum_{i} \mathrm{~d}_{i} g_{\varepsilon}\left(a_{i}\right) \delta_{a_{i}}\right|=|| \partial \Omega\left|-2 \pi \sum_{i} \mathrm{~d}_{i} g\left(a_{i}\right)\right| .
$$

Thus the measure curl $(g \nabla \varphi-u)\left\lfloor_{\Omega \backslash\left\{a_{i}\right\}}\right.$ plays the same role as the measure div $\left(\varphi u+u^{\perp}\right)$ in [30]. It carries a cost of the jumps of $u$ and $\varphi$ along the singular lines of $u$. But contrarily to the case of the $S^{1}$ constraint, some jumps of $2 \pi$ of $\varphi$ have now been removed by the presence of vortices.

This can be made rigorous in the case of prescribed vortices for example. Nevertheless, we are still able to get a rigorous result without any assumption on the vortices.

Let us introduce a few more notations. $g$ will be assumed to be nonnegative for simplicity (the general case is treated in Sect. 3). The total degree on the level-set $\left\{g_{\varepsilon}=t\right\}$ will be denoted by, i.e.

$$
D_{\varepsilon}(t)=\sum_{i / g_{\varepsilon}\left(a_{i}^{\varepsilon}\right) \geq t} \mathrm{~d}_{i}^{\varepsilon} \in \mathbb{Z},
$$

and

$$
M_{\varepsilon}(t)=2 \pi \int_{0}^{t} D_{\varepsilon}(s) \mathrm{d} s
$$

We also define

$$
p_{\varepsilon}(t)=\int_{\left\{g_{\varepsilon}=t\right\} \cap \Omega}\left|\nabla g_{\varepsilon}\right|
$$

(extended by 0 for $t \geq \max g_{\varepsilon}$ or $t \leq \min g_{\varepsilon}$ ). We denote

$$
p(t)=\mathcal{H}^{1}(\{g=t\})
$$

extended by the value 0 for $t>\max g$, and $|\partial \Omega|=\mathcal{H}^{1}(\partial \Omega)$ for $t<0$. In fact, $p$ is in $L^{1}$ and possibly has jumps. We will show $p_{\varepsilon} \rightarrow p$ in $L^{1}\left(\mathbb{R}^{+}\right)$. With an abuse of notation, we define the $B V$ norm of a function of $\mathbb{R}$ by

$$
\|f\|_{B V}=\int_{\mathbb{R}}\left|f^{\prime}\right|:=\sup _{\substack{\zeta \in C_{0}^{\infty}(\mathbb{R}) \\|\zeta| \leq 1}} \int_{\mathbb{R}} \zeta^{\prime} f .
$$


Theorem 3. Assume $E_{\varepsilon}\left(u^{\varepsilon}\right) \leq C, u^{\varepsilon} \rightarrow u$ in $\cap L^{p}(\Omega)$ and $g \geq 0$ in $\Omega$. Then, as $\varepsilon \rightarrow 0$,

$$
p_{\varepsilon}+M_{\varepsilon} \text { is bounded in } B V_{\mathrm{loc}}\left(\mathbb{R}^{+}\right) \text {. }
$$

Extracting subsequences if necessary, there exist $M \in L^{1}\left(\mathbb{R}^{+}\right)$and $D=\frac{M^{\prime}}{2 \pi}$ in $W^{-1,1}\left(\mathbb{R}^{+}\right)$such that

$$
p_{\varepsilon}+M_{\varepsilon} \rightarrow p+M \quad \text { in } B V_{\mathrm{loc}}\left(\mathbb{R}^{+}\right)
$$

i.e., denoting by $\mathcal{M}\left(\mathbb{R}^{+}\right)$the dual of $C_{0}^{0}\left(\mathbb{R}^{+}\right)$,

$$
p_{\varepsilon}^{\prime}+2 \pi D_{\varepsilon} \rightarrow p^{\prime}+2 \pi D \quad \text { in } \mathcal{M}\left(\mathbb{R}^{+}\right) .
$$

Moreover,

$$
\liminf _{\varepsilon \rightarrow 0} E_{\varepsilon}\left(u^{\varepsilon}\right) \geq E_{0}(u, D)=\|p+M\|_{B V\left(\mathbb{R}^{+}\right)}=\int_{0}^{+\infty}\left|p^{\prime}+2 \pi D\right| .
$$

This lower bound is obtained by integrating the quantity $\nabla^{\perp} g_{\varepsilon} \cdot \nabla \varphi_{\varepsilon}+\operatorname{curl} u^{\varepsilon}$ over regions $\left\{t \leq g_{\varepsilon} \leq t+\mathrm{d} t\right\}$. Thus we reduce to an integral over $\mathbb{R}$ : the integration variable $t$ is the value of $g$. Although it only gives information transversally to the level-sets of $g$, it appears to be a relevant variable. There does not seem to be an analogue global formulation available for e.g. the Aviles-Giga energy.

The limiting energy $E_{0}$ does not depend only on $u$, but on the couple $(u, D)$. It appears that it is the quantity $D(t)$, rather than the vortices themselves, which is relevant. In addition, let us point out that $D$ is not necessarily integer-valued, because vortices of opposite signs can accumulate (counter-examples can be easily constructed); $D$ is only a bounded measure.

If however limiting vortices $\left(a_{i}, d_{i}\right)$ can be defined, e.g. if they are fixed a priori, then $D \in \mathbb{Z}$ and $D(t)=$ $\sum_{i / g\left(a_{i}\right) \geq t} \mathrm{~d}_{i}$ and, going back to the lower bound (1.31), one may write

$$
\begin{aligned}
\int_{0}^{+\infty}\left|p^{\prime}+2 \pi D\right| & \geq\left|\int_{0}^{+\infty} p^{\prime}+2 \pi D\right|=\left|p(\infty)-p(0)+2 \pi \sum_{i} \mathrm{~d}_{i} g\left(a_{i}\right)\right| \\
& \geq|| \partial \Omega\left|-2 \pi \sum_{i} \mathrm{~d}_{i} g\left(a_{i}\right)\right|
\end{aligned}
$$

using the fact that $g=0$ on $\partial \Omega$ hence $p(0) \geq|\partial \Omega|$; hence one recovers the formula (1.23).

Conversely, taking $u=u_{*}=-\nabla^{\perp} g_{0}$ where $g_{0}=\operatorname{dist}(., \partial \Omega)$, and putting one vortex $a$ of degree 1 at a point of maximum of $g_{0}$, one would get equality in formula (1.32) and an energy $|\partial \Omega|-2 \pi g_{0}(a)=|\partial \Omega|-2 \pi \max _{\Omega} g$. It follows that

$$
\limsup _{\varepsilon \rightarrow 0} \min _{H^{1}\left(\Omega, S^{2}\right)} E_{\varepsilon} \leq|\partial \Omega|-|B|,
$$

where $|B|$ is the perimeter of a maximal disc $B$ inscribed in $\Omega$. This provides an upper bound which is already smaller than $|\partial \Omega|$, that of [30] and of pure $S^{1}$ configurations. If the domain is not convex, it is more interesting to put vortices of degree +1 at local maxima of $g_{0}$ and vortices of degree -1 at saddle points of $g_{0}$ in such a way that the total degree on each connected component of a level-set of $g$ is 1 .

The question of finding and describing an optimal configuration (which is probably not unique and depends on the geometry), i.e. one which minimizes the limiting energy $\int_{\Sigma_{u}} A(X)$, is open and seems difficult in general. This is another main difference with the purely $S^{1}$-case of [30], where $u_{*}$ was the minimizer. We refer to Section 4.1 for more examples and counter-examples. 
The paper is organized as follows: Section 2 contains the proof of Theorem 1, Section 3 the proof of Theorem 3. Both these sections are more analytical and may be skipped in a first reading. Section 4.1 contains examples and Section 4.2 the construction of optimal cross-tie wall profiles.

\section{The Limiting JUmp EnERGY}

We consider any family $u^{\varepsilon} \in H^{1}\left(\Omega, S^{2}\right)$ such that $E_{\varepsilon}\left(u^{\varepsilon}\right) \leq C$ and wish to prove convergence and lower bounds. Here, $\varepsilon$ will stand for any sequence $\varepsilon_{n}$ going to 0 .

Lemma 2.1. Let $u^{\varepsilon}$ be in $H^{1}\left(\Omega, S^{2}\right)$ such that $E_{\varepsilon}\left(u^{\varepsilon}\right) \leq C$. There exists $u \in \cap_{p<\infty} L^{p}\left(\Omega, S^{1}\right)$ and $g \in$ $\cap_{p<\infty} W_{0}^{1, p}(\Omega)$ such that (up to extraction)

$$
\begin{gathered}
u^{\varepsilon} \rightarrow u \quad \text { in } \cap_{p<\infty} L^{p}(\Omega) \\
\begin{cases}u=-\nabla^{\perp} g & \text { in } \Omega \quad|\nabla g|=1 \\
g=0 & \text { on } \partial \Omega\end{cases}
\end{gathered}
$$

(i.e. the limiting $u$ is a divergence-free, $S^{1}$-valued vector field tangent to $\partial \Omega$ ).

Proof. As written in the introduction (Eq. (1.5)), $H$ is defined by

$$
\left\{\begin{array}{l}
\partial_{1}\left(u_{1} \mathbf{1}_{\Omega}+H_{1}\right)+\partial_{2}\left(u_{2} \mathbf{1}_{\Omega}+H_{2}\right)=0 \\
\partial_{1} H_{2}-\partial_{2} H_{1}=0
\end{array}\right.
$$

and we have the standard estimate

$$
\forall 1<p<\infty, \exists C_{p} \quad\left\|H_{\varepsilon}\right\|_{L^{p}\left(\mathbb{R}^{2}\right)} \leq C\left\|u^{\varepsilon}\right\|_{L^{p}(\Omega)} \leq C_{p}
$$

Also $H_{\varepsilon} \rightarrow 0$ in $L^{2}\left(\mathbb{R}^{2}\right)$ hence $H_{\varepsilon} \rightarrow 0$ in $\cap_{p} L^{p}\left(\mathbb{R}^{2}\right)$.

We can write a two-dimensional Hodge decomposition of $u_{\|}^{\varepsilon} \mathbf{1}_{\Omega}\left(\right.$ where $u_{\|}=\left(u_{1}, u_{2}\right)$ ):

$$
u_{\|}^{\varepsilon} \mathbf{1}_{\Omega}=-H_{\varepsilon}-K_{\varepsilon}
$$

with $K_{\varepsilon}=\nabla^{\perp} g_{\varepsilon}$ a divergence-free vector-field in $\cap_{p<\infty} L^{p}\left(\mathbb{R}^{2}\right)$ and $\left\|K_{\varepsilon}\right\|_{L^{2}\left(\mathbb{R}^{2}\right)} \leq C,\left\|g_{\varepsilon}\right\|_{H^{1}\left(\mathbb{R}^{2}\right)} \leq C$. Then,

$$
\left\|\Delta g_{\varepsilon}\right\|_{L^{2}(\Omega)}=\left\|\operatorname{curl} u_{\|}^{\varepsilon}\right\|_{L^{2}(\Omega)} \leq C\left\|\nabla u^{\varepsilon}\right\|_{L^{2}(\Omega)} .
$$

Let now $\Omega^{\prime}$ be any open subset whose closure is in $\Omega$. By elliptic regularity,

$$
\left\|g_{\varepsilon}\right\|_{H^{2}\left(\Omega^{\prime}\right)} \leq C\left\|\nabla u^{\varepsilon}\right\|_{L^{2}(\Omega)} \leq \frac{C}{\sqrt{\varepsilon}}
$$

in view of the energy upper bound $E_{\varepsilon}\left(u^{\varepsilon}\right) \leq C$. Thus,

$$
\left\|\nabla K_{\varepsilon}\right\|_{L^{2}\left(\Omega^{\prime}\right)} \leq \frac{C}{\sqrt{\varepsilon}} .
$$

On the other hand,

$$
|| K_{\varepsilon}|-1|=|| H_{\varepsilon}+u_{\|}^{\varepsilon}\left|-\frac{\left|u_{\|}^{\varepsilon}\right|}{\left|u_{\|}^{\varepsilon}\right|}\right| \leq\left|H_{\varepsilon}\right|+\left|u_{\|}^{\varepsilon}-\frac{u_{\|}^{\varepsilon}}{\left|u_{\|}^{\varepsilon}\right|}\right|=\left|H_{\varepsilon}\right|+|| u_{\|}^{\varepsilon}|-1|,
$$


hence

$$
\left\|1-\left|K_{\varepsilon}\right|\right\|_{L^{2}(\Omega)} \leq\left\|H_{\varepsilon}\right\|_{L^{2}(\Omega)}+\left\|\left|u_{\|}^{\varepsilon}\right|-1\right\|_{L^{2}(\Omega)}
$$

But we also have

$$
\int_{\Omega}|| u_{\|}^{\varepsilon}|-1|^{2} \leq C \int_{\Omega}\left|u_{3}^{\varepsilon}\right|^{2} \leq C c_{\varepsilon} \leq C \varepsilon
$$

in view of the energy bound, so

$$
\left\|1-\left|K_{\varepsilon}\right|\right\|_{L^{2}(\Omega)} \leq C \sqrt{\varepsilon}
$$

Combining this with (2.5), we obtain

$$
\left\|\nabla K_{\varepsilon}\right\|_{L^{2}\left(\Omega^{\prime}\right)}\left\|1-\left|K_{\varepsilon}\right|\right\|_{L^{2}\left(\Omega^{\prime}\right)} \leq C
$$

while $\operatorname{div} K_{\varepsilon}=0$. The result of [25] allows us to conclude that $K_{\varepsilon}$ is compact in $\cap_{p<\infty} L^{p}\left(\Omega^{\prime}\right)$.

In the meantime, $H_{\varepsilon} \rightarrow 0$ in $\cap_{p<\infty} L^{p}\left(\mathbb{R}^{2}\right)$ hence from $(2.4), u_{\|}^{\varepsilon}$ is also compact in $\cap_{p<\infty} L^{p}\left(\Omega^{\prime}\right)$. From the energy bound $\int_{\Omega}\left|u^{\varepsilon}{ }_{3}\right|^{2} \rightarrow 0$ while $\left|u^{\varepsilon}{ }_{3}\right| \leq 1$, thus we can say that $\left\|u^{\varepsilon}\right\|_{L^{p}(\Omega)} \rightarrow 0$ for all $p<\infty$; consequently $u^{\varepsilon}$ is also compact in $\cap_{p<\infty} L^{p}\left(\Omega^{\prime}\right)$ and converges to some $u$. Then, up to extraction, $u^{\varepsilon} \rightarrow u$ a.e. in $\Omega$ and $\left|u^{\varepsilon}\right|$ is bounded by 1 , hence by Lebesgue's theorem $u^{\varepsilon} \rightarrow u$ in $L^{p}(\Omega)$. $u$ is $S^{1}$-valued because $u^{\varepsilon}{ }_{3} \rightarrow 0$ in $\cap_{p<\infty} L^{p}$ and thus a.e. Passing to the limit in (2.2), we get that

$$
\operatorname{div}\left(u \mathbf{1}_{\Omega}\right)=0 \quad \text { in } W^{-1, p}\left(\mathbb{R}^{2}\right)
$$

and thus we can find $g \in W^{1, \infty}\left(\mathbb{R}^{2}\right)$ such that $u \mathbf{1}_{\Omega}=-\nabla^{\perp} g$. The function $g$ will be constant in $\mathbb{R}^{2} \backslash \Omega$ hence can be chosen to be zero in $\mathbb{R}^{2} \backslash \Omega$. This proves the lemma.

We then have the following lemma, whose proof is postponed until the end of the section.

Lemma 2.2. Let $u^{\varepsilon}$ be such that $E_{\varepsilon}\left(u^{\varepsilon}\right) \leq C$. We can find $\tilde{u}^{\varepsilon} \in \cap_{1 \leq p<2} W^{1, p}\left(\Omega, S^{1}\right) \cap H^{1}\left(\Omega \backslash \cup_{i=1}^{n(\varepsilon)}\left\{a_{i}^{\varepsilon}\right\}, S^{1}\right)$ where for each $\varepsilon,\left\{a_{1}^{\varepsilon}, \cdots, a_{n(\varepsilon)}^{\varepsilon}\right\}$ is a finite set of points of $\Omega$; and satisfying the following:

$$
\begin{array}{cc}
\left\|\tilde{u}^{\varepsilon}-u^{\varepsilon}\right\|_{L^{p}(\Omega)} \rightarrow 0 & \forall p<\infty \\
\left\|\tilde{H}_{\varepsilon}-H_{\varepsilon}\right\|_{L^{p}(\Omega)} \rightarrow 0 & \forall p<\infty
\end{array}
$$

where $\tilde{H}_{\varepsilon}$ is the stray field generated by $\tilde{u}^{\varepsilon}$ according to (2.2), and

$$
E_{\varepsilon}\left(u^{\varepsilon}\right) \geq \int_{\Omega}\left|H_{\varepsilon}\right|\left|\nabla u_{\|}^{\varepsilon}\right|=\int_{\Omega}\left|\tilde{H}_{\varepsilon}\right|\left|\nabla \tilde{u^{\varepsilon}}\right|+o(1) .
$$

We then introduce notations for maps in $B V\left(\Omega, S^{1}\right)$. We recall the definition:

$$
B V\left(\Omega, S^{1}\right):=\left\{u \in B V\left(\Omega, \mathbb{R}^{2}\right), \quad u(x) \in S^{1} \text { a.e. } x \in \Omega\right\} .
$$

Recall that a map $u \in B V\left(\Omega, \mathbb{R}^{2}\right)$ is approximately continuous in the whole of $\Omega$ except on a 1-dimensional rectifiable subset of $\Omega$ called the jump set of $u$ that we will denote here by $\Sigma_{u}$ (see [18], Sect. 5.9).

For $\mathcal{H}^{1}$-a.e. $x \in \Sigma_{u}$ we denote by $\nu_{u}(x)$ the approximate unit normal for a given regular choice of orientation of the at most countable union of $C^{1}$ curves containing $\mathcal{H}^{1}$-almost the whole of $\Sigma_{u}$. Denote

$$
H_{\nu_{u}}^{ \pm}:=\left\{y \in \mathbb{R}^{2}, \quad(y-x) \cdot \nu_{u}(x) \geq 0(\text { resp. } \leq 0)\right\}
$$


Finally, recall that $\mathcal{H}^{1}$-a.e. point in $\Sigma_{u}$ admits a left and right approximate limit along $\Sigma_{u}$ : there exists two measurable functions $u_{+}$and $u_{-}$on $\Sigma_{u}$ such that for $\mathcal{H}^{1}$-a.e. point $x$ in $\Sigma_{u}$ we have

$$
\lim _{r \rightarrow 0} \frac{1}{r^{2}} \int_{B_{r}(x) \cap H_{\nu_{u}}^{ \pm}}\left|u(y)-u_{ \pm}(x)\right| \mathrm{d} y=0 .
$$

The following bound holds

$$
\int_{\Sigma_{u}}\left|u_{+}-u_{-}\right| \mathrm{d} \mathcal{H}^{1} \leq \sum_{i=1}^{2} \int_{\Sigma_{u}}\left|u_{+}^{i}-u_{-}^{i}\right| \mathrm{d} \mathcal{H}^{1} \leq \sum_{i=1}^{2}\left\|u_{i}\right\|_{B V} \leq \sqrt{2}\|u\|_{B V}
$$

Finally, We call $X$ the measurable function from $\Sigma_{u}$ into $[0, \pi / 2]$ given by

$$
\left.X=\frac{1}{2} \min \mid \widehat{\left(u_{ \pm}, u_{\mp}\right.}\right) \mid .
$$

We shall now establish the following result:

Theorem 1. Let $\varepsilon_{n} \rightarrow 0$ and $u^{\varepsilon_{n}}$ be sequences of maps from $\Omega$ into $S^{2}$ satisfying $E_{\varepsilon_{n}}\left(u^{\varepsilon_{n}}\right) \leq C$. Then, up to extraction, $u^{\varepsilon_{n}}$ converges in $\cap_{p<\infty} L^{p}(\Omega)$ to some limit $u$ which satisfies (1.7). If in addition $u \in B V\left(\Omega, S^{1}\right)$, then

$$
\liminf _{n \rightarrow \infty} E_{\varepsilon_{n}}\left(u^{\varepsilon_{n}}\right) \geq \int_{\Sigma_{u}} A(X) \mathrm{d} \mathcal{H}^{1}
$$

where

$$
\begin{aligned}
& A(X)=2|\sin X-X \cos X| \quad \text { for } X \in\left[0, \frac{\pi}{4}\right] \\
& A(X)=2\left|\left(X-\frac{\pi}{2}\right) \cos X-\sin X+\sqrt{2}\right| \text { for } X \in\left[\frac{\pi}{4}, \frac{\pi}{2}\right] .
\end{aligned}
$$

Proof. $\varepsilon_{n}$ will be simply denoted $\varepsilon$.

Step 1: Using the result of Lemma 2.2, we have

$$
E_{\varepsilon}\left(u^{\varepsilon}\right) \geq \int\left|\tilde{H}_{\varepsilon}\right|\left|\nabla \tilde{u}^{\varepsilon}\right|-o(1)
$$

We introduce

$$
\left\{\begin{array}{l}
g(s)=s \quad \text { for } s \in\left[-\frac{\pi}{4}, \frac{\pi}{4}\right] \\
g(s)=\frac{\pi}{2}-s \text { for } s \in\left[\frac{\pi}{4}, \frac{3 \pi}{4}\right]
\end{array}\right.
$$

and complete it to $\mathbb{R}$ into a continuous $\pi$-periodic function. Observe that $\left|g^{\prime}\right|=1$. We then define for $\sigma=\mathrm{e}^{i s_{0}} \in S^{1}$,

(again $\left|f^{\prime}\right|=1$ ), and the vector-field

$$
f_{\sigma}\left(\mathrm{e}^{i s}\right)=g\left(s-s_{0}\right)
$$

$$
V_{\sigma}(t)=\left(\begin{array}{l}
-\int_{s_{0}}^{t} g^{\prime}\left(\tau-s_{0}\right) \cos \tau \mathrm{d} \tau=-\int_{0}^{t-s_{0}} g^{\prime}(\tau) \cos \left(\tau+s_{0}\right) \mathrm{d} \tau \\
-\int_{s_{0}}^{t} g^{\prime}\left(\tau-s_{0}\right) \sin \tau \mathrm{d} \tau=-\int_{0}^{t-s_{0}} g^{\prime}(\tau) \sin \left(\tau+s_{0}\right) \mathrm{d} \tau
\end{array}\right) .
$$


Observe that $\int g^{\prime} \cos \left(\tau+s_{0}\right)$ and $\int g^{\prime} \sin \left(\tau+s_{0}\right)$ are $2 \pi$-periodic, therefore $V_{\sigma}$ is a $2 \pi$-periodic vector-field, hence can be considered as a map from $S^{1}$ to $\mathbb{R}^{2}$. In fact $f_{\sigma}$ and $V_{\sigma}$ are constructed so that for any smooth map $v$ into $S^{1}$ we have

$$
\operatorname{div}\left(V_{\sigma}(v)\right)+\nabla\left(f_{\sigma}(v)\right) \cdot v=0
$$

We claim that for any $\sigma=\mathrm{e}^{i s_{0}} \in S^{1}$, we have

$$
\operatorname{div}\left(f_{\sigma}\left(\tilde{u}^{\varepsilon}\right)\left(\tilde{u}^{\varepsilon}+\tilde{H}_{\varepsilon}\right)+V_{\sigma}\left(\tilde{u}^{\varepsilon}\right)\right)=\frac{f_{\sigma}^{\prime}\left(\tilde{u}^{\varepsilon}\right)}{i \tilde{u}^{\varepsilon}} \nabla \tilde{u}^{\varepsilon} \cdot \tilde{H}_{\varepsilon} \quad \text { in } \mathcal{D}^{\prime}(\Omega) .
$$

Then, assuming (2.15), since $\left|\frac{f_{\sigma}^{\prime}\left(\tilde{u}^{\varepsilon}\right)}{i \tilde{u}^{\varepsilon}}\right| \leq 1$, we deduce that

$$
\forall \sigma \in S^{1}, \quad E_{\varepsilon}\left(u^{\varepsilon}\right) \geq \int_{\Omega}\left|\nabla \tilde{u}^{\varepsilon}\right|\left|\tilde{H}_{\varepsilon}\right|-o(1) \geq \int_{\Omega}\left|\operatorname{div}\left(f_{\sigma}\left(\tilde{u}^{\varepsilon}\right)\left(\tilde{u}^{\varepsilon}+\tilde{H}_{\varepsilon}\right)+V_{\sigma}\left(\tilde{u}^{\varepsilon}\right)\right)\right|-o(1),
$$

which replaces the lower bound $\int_{\Omega}\left|\operatorname{div}\left(\varphi u+u^{\perp}\right)\right|$ in the $S^{1}$-valued case. Observe that it is also an analogue of the method introduced by Jin and Kohn in [22] to get lower bounds for the Aviles-Giga energy (1.9). The result will follow by passing to the limit $\varepsilon \rightarrow 0$.

Step 2: Proof of (2.15). Observe first that both sides of the equality have a meaning since, by construction (Lem. 2.1), $\tilde{H}_{\varepsilon} \in \cap_{p<+\infty} L^{p}$ and $\nabla \tilde{u}^{\varepsilon} \in L^{q}$ for any $q<2$. Because of (2.14), since smooth maps are strongly dense in $W^{1,2}\left(\Omega^{\prime}, S^{1}\right)$, where $\Omega^{\prime}$ is an open simply connected subset of $\Omega$ containing no vortices of $\tilde{u}^{\varepsilon}$, we deduce that (2.15) holds once we replace $\Omega$ by such an $\Omega^{\prime}$. Since $\tilde{u}^{\varepsilon}$ has only finitely many vortices $a_{1}^{\varepsilon} \cdots a_{n(\varepsilon)}^{\varepsilon}$, the distribution

$$
\operatorname{div}\left(f_{\sigma}\left(\tilde{u}^{\varepsilon}\right)\left(\tilde{u}^{\varepsilon}+\tilde{H}_{\varepsilon}\right)+V_{\sigma}\left(\tilde{u}^{\varepsilon}\right)\right)-f_{\sigma}^{\prime}\left(\tilde{u}^{\varepsilon}\right)\left(i \tilde{u}^{\varepsilon}\right)^{-1} \nabla \tilde{u}^{\varepsilon} \cdot \tilde{H}_{\varepsilon}
$$

supported at the isolated points $a_{j}^{\varepsilon}$ is a combination of $\partial^{k} \delta_{a_{j}}$ and since

$$
\operatorname{div}\left(f_{\sigma}\left(\tilde{u}^{\varepsilon}\right)\left(\tilde{u}^{\varepsilon}+\tilde{H}_{\varepsilon}\right)+V_{\sigma}\left(\tilde{u}^{\varepsilon}\right)\right) \in W^{-1, p}
$$

for any $1<p<+\infty$, it has to vanish and (2.15) is proved.

Step 3: Clean-up of $\Sigma_{u}$. Let $\delta>0$. The rectifiability property of $\Sigma_{u}$ means that $\Sigma_{u} \subset \cup_{k=1}^{+\infty} \Gamma^{k} \cup \Gamma^{0}$ where $\Gamma^{k}$ for $k \geq 1$ are $C^{1}$ embedded curves and $\mathcal{H}^{1}\left(\Gamma^{0}\right)=0$. Choose an integer $N$ such that

$$
\int_{\Sigma_{u} \cap \cup_{k=N+1}^{+\infty} \Gamma^{k}}\left|u_{+}-u_{-}\right| \mathrm{d} \mathcal{H}^{1} \leq \delta
$$

We may always assume that $\left(\bar{\Gamma}^{k}\right)_{k=1 \cdots N}$ is made of disjoint embedded $C^{1}$ curves. Since $u$ is left and right approximately continuous $\mathcal{H}^{1}$ a.e. on $\Sigma_{u}$, we may extend the $\mathcal{H}^{1}$-measurable functions $u_{+}$and $u_{-}$on $\Sigma_{u} \cap$ $\cup_{k=1 \cdots N} \bar{\Gamma}^{k}$ by the approximate limit of $u$ on the whole of $\cup_{k=1 \cdots N} \bar{\Gamma}^{k}$.

On $\cup_{k=1 \cdots N} \bar{\Gamma}^{k}$ we consider the subset $L^{N}$ of Lebesgue points for $u_{+}$and $u_{-}\left(\mathcal{H}^{1}\left(\cup_{k=1 \cdots_{N}} \bar{\Gamma}^{k} \backslash L^{N}\right)=0\right)$. Let $E^{N}$ be a compact subset of $L^{N}$ such that

$$
\int_{E^{N}}\left|u_{+}-u_{-}\right| \mathrm{d} \mathcal{H}^{1} \geq \int_{\cup_{k=1 \ldots N} \bar{\Gamma}^{k}}\left|u_{+}-u_{-}\right| \mathrm{d} \mathcal{H}^{1}-\delta .
$$


The existence of such a compact set for the given Radon measure $\left|u_{+}-u_{-}\right| d \mathcal{H}^{1}\left\lfloor L^{N}\right.$ is given by the standard approximation of measurable sets by compact sets (see for instance [18], Sect. 1.1). To any $x \in E^{N}$ we assign a positive radius $\rho_{x}$ such that

$$
\frac{1}{\left|\cup_{k=1 \cdots N} \bar{\Gamma}^{k} \cap B_{\rho_{x}}(x)\right|} \int_{\cup_{k=1 \cdots N} \bar{\Gamma}^{k} \cap B_{\rho_{x}}(x)}\left|u_{ \pm}(y)-u_{ \pm}(x)\right| \mathrm{d} \mathcal{H}^{1} \leq \frac{\delta}{\mathcal{H}^{1}\left(\cup_{k=1}^{N} \bar{\Gamma}^{k}\right)}
$$

(by definition of a Lebesgue point). From $\left(B_{\rho_{x}}(x)\right)_{x \in E^{N}}$ we may extract a Besicovitch subcovering of $E^{N}$ $\left(B_{\rho_{j}}\left(x_{j}\right)\right)$ made of at most countably many balls, each of them intersecting at most $C_{0}$ other balls, where $C_{0}$ is a universal number (see [18], Sect. 1.5.2). Observe that here this covering has to be finite since $E^{N}$ is compact. Consider a partition of unity $\left(\phi_{j}\right)_{j}$ modelled on this subcovering so that $\phi_{j} \in C_{0}^{\infty}\left(B_{\rho_{j}}\left(x_{j}\right)\right), \phi_{j} \geq 0$ and $\sum_{j} \phi_{j} \equiv 1$ on $E^{N}$, and $\pi$ the projection from a tubular neighborhood of $\cup_{k} \bar{\Gamma}^{k}$ to $\cup_{k} \bar{\Gamma}^{k}$. We thus have reduced to the case of a smooth curve.

Step 4: We choose $\sigma$ to be the unit normal $\nu\left(x_{j}\right)$ at $x_{j}$ to the oriented set of curves $\cup_{k} \bar{\Gamma}^{k}$ so that $\nu\left(x_{j}\right)$ belongs to the smallest arc joining $u_{+}\left(x_{j}\right)$ and $u_{-}\left(x_{j}\right)$. Then, from (2.16),

$$
E_{\varepsilon}\left(u_{\varepsilon}\right) \geq \sum_{j} \int_{\Omega} \phi_{j}(\pi(x))\left|\operatorname{div}\left(f_{\nu\left(x_{j}\right)}\left(\tilde{u}^{\varepsilon}\right)\left(\tilde{u}^{\varepsilon}+\tilde{H}_{\varepsilon}\right)+V_{\nu\left(x_{j}\right)}\left(\tilde{u}^{\varepsilon}\right)\right)\right|-o(1) .
$$

Since $\tilde{u^{\varepsilon}} \rightarrow u$ in $\cap_{p} L^{p}(\Omega)$,

$$
f_{\nu\left(x_{j}\right)}\left(\tilde{u}^{\varepsilon}\right)\left(\tilde{u}^{\varepsilon}+\tilde{H}_{\varepsilon}\right)+V_{\nu\left(x_{j}\right)}\left(\tilde{u}^{\varepsilon}\right) \rightarrow f_{\nu\left(x_{j}\right)}(u) u+V_{\nu\left(x_{j}\right)}(u) \text { in } \cap_{p} L^{p} .
$$

Thus,

$$
\liminf _{\varepsilon \rightarrow 0} E_{\varepsilon}\left(u^{\varepsilon}\right) \geq \sum_{j} \int_{\Omega} \phi_{j}(\pi(x))\left|\operatorname{div}\left(f_{\nu\left(x_{j}\right)}(u) u+V_{\nu\left(x_{j}\right)}(u)\right)\right| .
$$

Since $u$ is assumed to be $B V$, and $\operatorname{div} u=0$, by the $B V$ chain rule of Volpert, we find that $\operatorname{div}\left(f_{\nu\left(x_{j}\right)}(u) u+V_{\nu\left(x_{j}\right)}(u)\right)$ is supported only in $\Sigma_{u}$ (see $\left.(2.14)\right)$ and

$\operatorname{div}\left(f_{\nu\left(x_{j}\right)}(u) u+V_{\nu\left(x_{j}\right)}(u)\right)$

$$
=\left(f_{\nu\left(x_{j}\right)}\left(u^{+}(x)\right) u^{+}(x)+V_{\nu\left(x_{j}\right)}\left(u^{+}(x)\right)-f_{\nu\left(x_{j}\right)}\left(u^{-}(x)\right) u^{-}(x)-V_{\nu\left(x_{j}\right)}\left(u^{-}(x)\right) u^{-}(x)\right) \cdot \nu(x) \mathcal{H}^{1}\left\lfloor\Sigma_{u} .\right.
$$

But in view of (2.20),

$$
\begin{aligned}
\sum_{j} \int_{\Sigma_{u}} \phi_{j}(\pi(x)) \mid\left(f_{\nu\left(x_{j}\right)}\left(u^{ \pm}(x)\right) u^{ \pm}(x)+V_{\nu\left(x_{j}\right)}\left(u^{ \pm}(x)\right)\right) \cdot \nu(x) & \\
& -\left(f_{\nu\left(x_{j}\right)}\left(u^{ \pm}(x)\right) u^{ \pm}(x)+V_{\nu\left(x_{j}\right)}\left(u^{ \pm}(x)\right)\right) \cdot \nu\left(x_{j}\right) \mid \leq C \delta .
\end{aligned}
$$

On the other hand, a simple computation yields the crucial relation

$$
\begin{aligned}
\mid\left(f_{\nu\left(x_{j}\right)}\left(u^{+}\right)\left(x_{j}\right) u^{+}\left(x_{j}\right)+V_{\nu\left(x_{j}\right)}\left(u^{+}\right)\right. & \left.\left(x_{j}\right)\right) \cdot \nu\left(x_{j}\right) \\
& -\left(f_{\nu\left(x_{j}\right)}\left(u^{-}\right)\left(x_{j}\right) u^{-}\left(x_{j}\right)+V_{\nu\left(x_{j}\right)}\left(u^{-}\right)\left(x_{j}\right)\right) \cdot \nu\left(x_{j}\right) \mid=A\left(X\left(x_{j}\right)\right) .
\end{aligned}
$$

Indeed, in the orthonormal frame to $\Sigma_{u}$ at $x_{j}$, the situation is that of a jump from $-X\left(x_{j}\right)$ to $X\left(x_{j}\right)$ with $\nu\left(x_{j}\right)=\mathrm{e}^{i 0}$. If $X \leq \frac{\pi}{4}, f\left(u_{ \pm}\right)= \pm X$ and $V\left(u_{ \pm}\right)=u_{ \pm}^{\perp}$ with $u_{ \pm}=(\cos X, \pm \sin X)$, hence the jump is

$$
X \cos X-\sin X+X \cos X-\sin X=2(X \cos X-\sin X) .
$$


If $X \in\left[\frac{\pi}{4}, \frac{\pi}{2}\right]$, then $f\left(u_{+}\right)=\frac{\pi}{2}-X, f\left(u_{-}\right)=-\frac{\pi}{2}+X, V\left(u_{+}\right)=-u_{+}^{\perp}+(-\sqrt{2}, \sqrt{2}), V\left(u_{-}\right)=-u_{-}^{\perp}+(\sqrt{2},-\sqrt{2})$ and the cost is

Combining (2.22-2.25), we obtain that

$$
2\left(\frac{\pi}{2}-X\right) \cos X+2(\sin X-\sqrt{2}) .
$$

$$
\liminf _{\varepsilon \rightarrow 0} E_{\varepsilon}\left(u_{\varepsilon}\right) \geq \int_{E^{N}} A(X)-C \delta
$$

and using (2.19) and (2.18) we obtain the result.

We finish with the

Proof of Lemma 2.2. We recall that we assume $c_{\varepsilon} \leq \varepsilon^{1+\delta}$ for some $\delta>0$ arbitrarily small. For any $\alpha \in S^{2}$ such that $\alpha_{3}>\frac{3}{4}$ we define a map $\pi_{a}$ from $S^{2}$ to $S^{1}$ as follows:

$$
\mid \begin{array}{ll}
\text { if }\left|x_{3}\right| \leq \frac{1}{4} & \pi_{\alpha}(x)=\left(\frac{x_{1}}{\left(x_{1}^{2}+x_{2}^{2}\right)^{\frac{1}{2}}}, \frac{x_{2}}{\left(x_{1}^{2}+x_{2}^{2}\right)^{\frac{1}{2}}}\right) \\
\text { if } x_{3} \geq \frac{1}{2}, x \neq \alpha & \begin{array}{ll}
\pi_{\alpha} \text { is the intersection with the equator of the unique geodesic joining } \\
\alpha \text { to the equator and containing } x
\end{array} \\
\begin{array}{ll}
\text { if } x_{3} \leq-\frac{1}{2}, x \neq-\alpha & \pi_{\alpha} \text { is the intersection with the equator of the unique geodesic joining } \\
& -\alpha \text { to the equator and containing } x .
\end{array}
\end{array}
$$

For $\frac{1}{4} \leq\left|x_{3}\right| \leq \frac{1}{2}, \pi_{\alpha}$ is extended in such a way that the following holds :

$$
\begin{gathered}
\text { for } x \neq \alpha,-\alpha, \quad\left|\nabla \pi_{\alpha}(x)\right| \leq \frac{C}{\inf (|x-\alpha|,|x+\alpha|)} \\
\left|x-\pi_{\alpha}(x)\right| \leq C x_{3} .
\end{gathered}
$$

Let $p<2$. For almost every $\alpha$ such that $\alpha_{3}>\frac{3}{4}, \alpha$ is a regular value of $u^{\varepsilon}$ i.e. $\left(u^{\varepsilon}\right)^{-1}(\alpha)$ is a finite set of points where the Jacobian determinant of $u^{\varepsilon}$ does not vanish.

Using (2.28), and $\omega$ being a subset of $\Omega$,

$$
\begin{aligned}
\int_{\alpha_{3}>\frac{3}{4}} \int_{\omega}\left|\nabla\left(\pi_{\alpha}\left(u^{\varepsilon}(x)\right)\right)\right|^{p} \mathrm{~d} x \mathrm{~d} \alpha & \leq C \int_{\alpha_{3}>\frac{3}{4}} \int_{\omega}\left|D \pi_{\alpha}\left(u^{\varepsilon}\right)\right|^{p}\left|\nabla u^{\varepsilon}\right|^{p}(x) \mathrm{d} x \mathrm{~d} \alpha \\
& \leq C \int_{\alpha_{3}>\frac{3}{4}} \int_{\omega} \frac{\left|\nabla u^{\varepsilon}\right|^{p}}{\left|u^{\varepsilon}-\alpha\right|^{p}}
\end{aligned}
$$

Using Fubini's theorem, this implies that

$$
\begin{aligned}
\int_{\alpha_{3}>\frac{3}{4}} \int_{\omega}\left|\nabla\left(\pi_{\alpha}\left(u^{\varepsilon}(x)\right)\right)\right|^{p} \mathrm{~d} x \mathrm{~d} \alpha & \leq C \int_{\omega}\left|\nabla u^{\varepsilon}(x)\right|^{p}\left(\int_{\alpha \in S^{2}} \frac{\mathrm{d} \alpha}{\left|u^{\varepsilon}(x)-\alpha\right|^{p}}\right) \mathrm{d} x \\
& \leq C \int_{\omega}\left|\nabla u^{\varepsilon}\right|^{p}
\end{aligned}
$$

(Indeed, since $p<2, \int_{\alpha \in S^{2}} \frac{\mathrm{d} \alpha}{|y-\alpha|^{p}}$ converges for every $y \in S^{2}$.) Using the mean-value theorem, it follows that there exists $\alpha^{\varepsilon} \in S^{2}$ such that $\alpha_{3}^{\varepsilon}>\frac{3}{4}$ and

$$
\int_{\omega}\left|\nabla\left(\pi_{\alpha}\left(u^{\varepsilon}\right)\right)\right|^{p} \leq C \int_{\omega}\left|\nabla u^{\varepsilon}\right|^{p}
$$


and such that $\left(u^{\varepsilon}\right)^{-1}\left(\alpha^{\varepsilon}\right)$ is a finite set. We then set

$$
\tilde{u^{\varepsilon}}(x)=\pi_{\alpha}\left(u^{\varepsilon}(x)\right) \text {. }
$$

$\tilde{u^{\varepsilon}}$ is $S^{1}$-valued and has the same regularity as $u^{\varepsilon}$ i.e. $H^{1}$ except for $x \in\left(u^{\varepsilon}\right)^{-1}\left(\alpha^{\varepsilon}\right)$. We denote by $a_{1}, \cdots, a_{n(\varepsilon)}^{\varepsilon}$ the set of singular points of $\tilde{u}^{\varepsilon}$ i.e. $\left(u^{\varepsilon}\right)^{-1}\left(\alpha^{\varepsilon}\right)$. Since $\tilde{u}^{\varepsilon}$ is $H^{1}$ away from these points, $\mathrm{d}_{i}^{\varepsilon}$, the degree of $\tilde{u}^{\varepsilon}$ at $a_{i}^{\varepsilon}$ is well-defined. Moreover, as soon as $\left|u^{\varepsilon}{ }_{3}(x)\right| \leq \frac{1}{4}, \tilde{u^{\varepsilon}}=\frac{u_{\|}^{\varepsilon}(x)}{\left|u_{\|}^{\varepsilon}(x)\right|}\left(u_{\|}\right.$denotes $\left.\left(u_{1}, u_{2}\right)\right)$ hence $\tilde{u^{\varepsilon}}$ has the same degree as $\tilde{u}^{\varepsilon}=\frac{u_{\|}^{\varepsilon}(x)}{\left|u_{\|}^{\varepsilon}(x)\right|}$ outside of the set $\left\{\left|u^{\varepsilon}{ }_{3}(x)\right| \geq \frac{1}{4}\right\}$, which is a small set: in view of the energy bound $\frac{1}{c_{\varepsilon}} \int_{\Omega}\left|u^{\varepsilon}\right|^{2} \leq C$, we have

$$
\operatorname{vol}\left(\left\{\left|u^{\varepsilon}{ }_{3}(x)\right| \geq \frac{1}{4}\right\}\right) \leq O\left(c_{\varepsilon}\right)
$$

Let us prove that for some $p>1$, we have

$$
\int_{\Omega}\left|\nabla\left(\tilde{u^{\varepsilon}}-u_{\|}^{\varepsilon}\right)\right|^{p} \rightarrow 0
$$

Indeed, in view of (2.30),

$$
\int_{\left|u^{\varepsilon}{ }_{3}\right| \geq \frac{1}{4}}\left|\nabla\left(\tilde{u}^{\varepsilon}-u_{\|}^{\varepsilon}\right)\right|^{p} \leq C \int_{\left|u^{\varepsilon}\right|^{3} \mid \geq \frac{1}{4}}\left|\nabla \tilde{u}^{\varepsilon}\right|^{p}+\left|\nabla u^{\varepsilon}\right|^{p} \leq C \int_{\mid u^{\varepsilon} \varepsilon^{2} \geq \frac{1}{4}}\left|\nabla u^{\varepsilon}\right|^{p} .
$$

Using Hölder's inequality,

$$
\int_{\left|u^{\varepsilon} 3\right| \geq \frac{1}{4}}\left|\nabla u^{\varepsilon}\right|^{p} \leq \operatorname{vol}\left(\left\{\left|u^{\varepsilon}(x)\right| \leq \frac{1}{4}\right\}\right)^{1-\frac{p}{2}}\left\|\nabla u^{\varepsilon}\right\|_{L^{2}(\Omega)}^{p} .
$$

But in view of the energy bound, we have $\left\|\nabla u^{\varepsilon}\right\|_{L^{2}(\Omega)} \leq \frac{C}{\varepsilon^{\frac{1}{2}}}$ and, using (2.32), we are led to

$$
\int_{\mid u^{\varepsilon_{3} \mid \geq \frac{1}{4}}}\left|\nabla u^{\varepsilon}\right|^{p} \leq O\left(\frac{c_{\varepsilon}^{1-\frac{p}{2}}}{\varepsilon^{\frac{p}{2}}}\right) .
$$

Since $c_{\varepsilon} \leq \varepsilon^{1+\delta}$, this implies that we can choose $p-1>0$ small enough compared to $\delta$ so that

$$
\int_{\mid u^{\varepsilon_{3} \mid \geq \frac{1}{4}}}\left|\nabla\left(\tilde{u}^{\varepsilon}-u_{\|}^{\varepsilon}\right)\right|^{p} \leq o(1)
$$

Next, we bound $\int_{\left|u^{\varepsilon} 3\right| \leq \frac{1}{4}}\left|\nabla\left(\tilde{u}^{\varepsilon}-u_{\|}^{\varepsilon}\right)\right|^{p}$. Recalling that $\tilde{u}^{\varepsilon}=\frac{u_{\|}^{\varepsilon}(x)}{\left|u_{\|}^{\varepsilon}(x)\right|}$ in the set $\left\{\left|u^{\varepsilon}{ }_{3}\right| \leq \frac{1}{4}\right\}$, we have

$$
\left|\nabla\left(u^{\tilde{\varepsilon}_{1}}-u_{1}^{\varepsilon}{ }_{1}\right)\right|=\left|\nabla\left(\frac{u^{\varepsilon}{ }_{1}}{\sqrt{\left(u^{\varepsilon} 1\right)^{2}+\left(u^{\varepsilon}\right)^{2}}}-u_{1}^{\varepsilon}\right)\right|=\left|\nabla\left(u_{1}^{\varepsilon}\left(\frac{1}{\sqrt{1-\left(u^{\varepsilon}\right)^{2}}}-1\right)\right)\right| .
$$

We observe that in $\left\{\left|u^{\varepsilon}{ }_{3}\right| \leq \frac{1}{4}\right\}$,

$$
\left|\frac{1}{\sqrt{1-\left(u^{\varepsilon} 3\right)^{2}}}-1\right| \leq C\left|u^{\varepsilon}{ }_{3}\right|^{2} \text { and }\left|\nabla \frac{1}{\sqrt{1-\left(u^{\varepsilon} 3\right)^{2}}}\right| \leq C\left|u^{\varepsilon}{ }_{3}\right|\left|\nabla u^{\varepsilon}{ }_{3}\right| .
$$


Thus, in $\left\{\left|u^{\varepsilon}{ }_{3}\right| \leq \frac{1}{4}\right\}$,

$$
\left|\nabla\left(u^{\tilde{\varepsilon}_{1}}-u^{\varepsilon}{ }_{1}\right)\right| \leq C\left(\left|u^{\varepsilon}{ }_{3}\right|^{2}\left|\nabla u_{1}^{\varepsilon}\right|+\left|u^{\varepsilon}{ }_{3}\right|\left|\nabla u^{\varepsilon}{ }_{3}\right|\right) \leq C\left|u^{\varepsilon}{ }_{3}\right|\left|\nabla u^{\varepsilon}\right| .
$$

Similarly, $\left|\nabla\left(u_{2}^{\varepsilon}-u^{\tilde{\varepsilon}_{2}}\right)\right| \leq C\left|u^{\varepsilon}{ }_{3}\right|\left|\nabla u^{\varepsilon}\right|$. Hence,

$$
\begin{aligned}
\int_{\left|u^{\varepsilon} 3\right| \leq \frac{1}{4}}\left|\nabla\left(\tilde{u^{\varepsilon}}-u_{\|}^{\varepsilon}\right)\right|^{p} & \leq C \int_{\Omega}\left|u^{\varepsilon}\right|_{3}^{p}\left|\nabla u^{\varepsilon}\right|^{p} \\
& \leq C\left(\int_{\Omega}\left|u^{\varepsilon}{ }_{3}\right|^{2}\right)^{1-\frac{p}{2}}\left(\int_{\Omega}\left|\nabla u^{\varepsilon}\right|^{2}\right)^{\frac{p}{2}} \\
& \leq C \frac{c_{\varepsilon}^{1-\frac{p}{2}}}{\varepsilon^{\frac{p}{2}}}
\end{aligned}
$$

where we have used again Hölder's inequality and the energy upper bound. Choosing again $p-1>0$ small enough, we can ensure (combining (2.34) and (2.35)) that (2.33) holds.

Then, from (2.29), we have for $2 \leq q<\infty$,

$$
\int_{\Omega}\left|\tilde{u}^{\varepsilon}-u^{\varepsilon}\right|^{q} \leq C \int_{\Omega}\left|u^{\varepsilon}\right|^{q} \leq C \int_{\Omega}\left|u^{\varepsilon}\right|^{2} \leq O\left(c_{\varepsilon}\right) .
$$

Hence, for all $2 \leq q<\infty,\left\|\tilde{u^{\varepsilon}}-u^{\varepsilon}\right\|_{L^{q}(\Omega)} \leq O\left(c_{\varepsilon}^{q}\right)$ which proves (2.8). In addition, we deduce the estimate

$$
\left\|H_{\varepsilon}-\tilde{H}_{\varepsilon}\right\|_{L^{q}\left(\mathbb{R}^{2}\right)} \leq C\left\|\tilde{u^{\varepsilon}}-u^{\varepsilon}\right\|_{L^{q}(\Omega)} \leq O\left(c_{\varepsilon}^{q}\right)
$$

where $\tilde{H}_{\varepsilon}$ is the demagnetizing field induced by $\tilde{u}^{\varepsilon}$ (see (2.2)). Thus, in view of (2.33),

$$
\int_{\Omega}\left|\nabla\left(\tilde{u}^{\varepsilon}-u_{\|}^{\varepsilon}\right)\right|\left|\tilde{H}_{\varepsilon}\right| \leq\left\|\nabla\left(\tilde{u}^{\varepsilon}-u_{\|}^{\varepsilon}\right)\right\|_{L^{p}(\Omega)}\left\|\tilde{H}_{\varepsilon}\right\|_{L^{q}\left(\mathbb{R}^{2}\right)} \leq C\left\|\nabla\left(\tilde{u}^{\varepsilon}-u_{\|}^{\varepsilon}\right)\right\|_{L^{p}(\Omega)}=o(1)
$$

while, using (2.36) and the energy bound

$$
\int_{\Omega}\left|\nabla u_{\|}^{\varepsilon}\right|\left|H_{\varepsilon}-\tilde{H}_{\varepsilon}\right| \leq\left\|\nabla u^{\varepsilon}\right\|_{L^{2}(\Omega)}\left\|H_{\varepsilon}-\tilde{H}_{\varepsilon}\right\|_{L^{2}} \leq C\left(\frac{c_{\varepsilon}}{\varepsilon}\right)^{\frac{1}{2}} \rightarrow 0 .
$$

We conclude that

$$
\int_{\Omega}\left|\nabla u_{\|}^{\varepsilon}\right|\left|H_{\varepsilon}\right|=\int_{\Omega}\left|\nabla \tilde{u}^{\varepsilon}\right|\left|\tilde{H}_{\varepsilon}\right|+o(1)
$$

Remark 2.1. It is not possible to define vortices of $u^{\varepsilon}$ directly because, for example, $u^{\varepsilon}{ }_{3}$ can be equal to 1 on a subset of $\Omega$ of nonempty interior. The construction of $\tilde{u}^{\varepsilon}$ allows to isolate points next to which $u^{\varepsilon}$ passes through the North or South pole, the vortices which are relevant being those with nonzero degree, i.e. those which are topological. This definition of vortices (and the choice of $\tilde{u}^{\varepsilon}$ ) may not seem intrinsic since they depend on a choice of the point $\alpha^{\varepsilon}$. However, the vortex-locations can only vary very little in space (on an $o(1)$ scale) and their degree is intrinsic.

\section{LOWER BOUNDS INVOLVING THE VORTICES}

Let $u^{\varepsilon} \in H^{1}\left(\Omega, S^{2}\right)$ be such that $E_{\varepsilon}\left(u^{\varepsilon}\right) \leq C$ and $u^{\varepsilon} \rightarrow u$ in $\cap_{p} L^{p}$. With Lemma 2.2, we may use (2.10) to start bounding the energy from below and hence, replacing $u^{\varepsilon}$ by $\tilde{u}^{\varepsilon}$, work on $\int_{\Omega}\left|\tilde{H}_{\varepsilon}\right|\left|\nabla \tilde{u}^{\varepsilon}\right|$. Then, we can define $g_{\varepsilon} \in \cap_{p<\infty} W^{1, p}\left(\mathbb{R}^{2}\right)$ such that

$$
\tilde{u}^{\varepsilon} \mathbf{1}_{\Omega}+\tilde{H}_{\varepsilon}=-\nabla^{\perp} g_{\varepsilon}
$$


Since $u^{\varepsilon} \rightarrow u$ strongly in $\cap_{p<\infty} L^{p}(\Omega)$ and $H_{\varepsilon} \rightarrow 0$ too, in view of (2.8) and (2.9) we have $\tilde{u}^{\varepsilon} \rightarrow u$ strongly in $\cap_{p<\infty} L^{p}(\Omega)$ and $\tilde{H}_{\varepsilon} \rightarrow 0$ in $\cap_{p<\infty} L^{p}\left(\mathbb{R}^{2}\right)$. Therefore, $g_{\varepsilon} \rightarrow g$ (defined in (2.1)) strongly in $\cap_{p<\infty} W^{1, p}\left(\mathbb{R}^{2}\right.$ ) (hence uniformly). From now on, we will assume that $u^{\varepsilon}$ has been replaced by $\tilde{u}^{\varepsilon} \in H^{1}\left(\Omega \backslash \cup_{i=1}^{n(\varepsilon)}\left\{a_{i}^{\varepsilon}\right\}, S^{1}\right)$ and write $u^{\varepsilon}$ instead of $\tilde{u}^{\varepsilon}, H_{\varepsilon}$ instead of $\tilde{H}_{\varepsilon}$. We thus have a family of $S^{1}$-valued vector fields with a finite number of singular points or "vortices" (for fixed $\varepsilon$ ). Since $u^{\varepsilon}$ is in $H^{1}$ away from these points, we can define its degree around $a_{i}^{\varepsilon}$, and write

$$
d_{i}^{\varepsilon}=\operatorname{deg}\left(u^{\varepsilon}, a_{i}^{\varepsilon}\right)
$$

Locally in $\Omega \backslash\left\{a_{1}^{\varepsilon}, \cdots, a_{n(\varepsilon)}^{\varepsilon}\right\}$, from the result of [8], $u^{\varepsilon}$ admits an $H^{1}$-lifting $\varphi_{\varepsilon}$ and we may define the vector-field

$$
V_{\varepsilon}=\left\langle\nabla u^{\varepsilon}, i u^{\varepsilon}\right\rangle "=" \nabla \varphi_{\varepsilon}
$$

which is well-defined a.e. in $\Omega$, curl-free in $\Omega \backslash\left\{a_{1}^{\varepsilon}, \cdots, a_{n(\varepsilon)}^{\varepsilon}\right\}$. As in [30], the lower bound of the energy will follow from bounding $\int_{\Omega}\left|H_{\varepsilon} \cdot V_{\varepsilon}\right|$.

In a first step, we shall assume that $g$ defined in (2.1) has a distinguished sign in $\Omega$, i.e., without loss of generality, that

We define

$$
\left\{\begin{array}{l}
g \geq 0 \text { in } \Omega \\
g=0 \text { on } \partial \Omega
\end{array}\right.
$$

$$
D_{\varepsilon}(t)=\frac{1}{2 \pi} \int_{\left\{g_{\varepsilon}=t\right\} \cap \Omega} V_{\varepsilon} \cdot \tau=\sum_{i / g_{\varepsilon}\left(a_{i}^{\varepsilon}\right) \geq t} \mathrm{~d}_{i}^{\varepsilon} \in \mathbb{Z}
$$

where the normal-vector $n$ to $\left\{g_{\varepsilon}=t\right\}$ is oriented towards the increasing $g_{\varepsilon}$. The function $D_{\varepsilon}$ is integer-valued on $\mathbb{R}^{+}$, equal to the total degree on the level-set $\left\{g_{\varepsilon}=t\right\}$. It is defined almost everywhere (for $t$ such that $\left\{g_{\varepsilon}=t\right\}$ is regular and does not contain any vortex-point). It can be defined for all $t$ through the formula $\sum_{i / g_{\varepsilon}\left(a_{i}^{\varepsilon}\right) \geq t} \mathrm{~d}_{i}^{\varepsilon}$ and extended by 0 for $t<0$. Then, we recall that

$$
M_{\varepsilon}(t)=2 \pi \int_{0}^{t} D_{\varepsilon}(s) \mathrm{d} s
$$

and $p_{\varepsilon}$ and $p$ are defined in (1.26) and (1.27). We have the following result:

\section{Lemma 3.1.}

$$
p_{\varepsilon} \rightarrow p \quad \text { in } L^{1}\left(\mathbb{R}^{+}\right) .
$$

Before proving this lemma, let us state the main result.

Theorem 3. Assume $E_{\varepsilon}\left(u^{\varepsilon}\right) \leq C, u^{\varepsilon} \rightarrow u$ in $\cap L^{p}(\Omega)$ and $g \geq 0$ in $\Omega$. Then, as $\varepsilon \rightarrow 0$,

$$
p_{\varepsilon}+M_{\varepsilon} \text { is bounded in } B V_{\mathrm{loc}}\left(\mathbb{R}^{+}\right) \text {. }
$$

Extracting subsequences if necessary, there exist $M \in L^{1}\left(\mathbb{R}^{+}\right)$and $D=\frac{M^{\prime}}{2 \pi}$ in $W^{-1,1}\left(\mathbb{R}^{+}\right)$such that

$$
p_{\varepsilon}+M_{\varepsilon} \rightarrow p+M \quad \text { in } B V_{\mathrm{loc}}\left(\mathbb{R}^{+}\right)
$$

i.e., denoting by $\mathcal{M}\left(\mathbb{R}^{+}\right)$the dual of $C_{0}^{0}\left(\mathbb{R}^{+}\right)$,

$$
p_{\varepsilon}^{\prime}+2 \pi D_{\varepsilon} \rightarrow p^{\prime}+2 \pi D \quad \text { in } \mathcal{M}\left(\mathbb{R}^{+}\right) .
$$


Moreover,

$$
\liminf _{\varepsilon \rightarrow 0} E_{\varepsilon}\left(u^{\varepsilon}\right) \geq E_{0}(u, D)=\|p+M\|_{B V\left(\mathbb{R}^{+}\right)}=\int_{0}^{+\infty}\left|p^{\prime}+2 \pi D\right|
$$

If in addition we assume that $u^{\varepsilon}$ does not have vortices in a set of the form $\{g \leq \delta\}$ for some $\delta>0$ and has a lifting $\varphi_{\varepsilon}$ in that set uniformly bounded in $L^{\infty}$, then the lower bound can be improved as follows:

$$
\liminf _{\varepsilon \rightarrow 0} E_{\varepsilon}\left(u^{\varepsilon}\right) \geq E_{0}(u, D)=\|p+M\|_{B V(\mathbb{R})}=\int_{\mathbb{R}}\left|p^{\prime}+2 \pi D\right|
$$

( $D$ being extended by 0 on $\mathbb{R}^{-}$and $p$ by $|\partial \Omega|$ ).

The result (3.9) is a stronger lower bound than (3.8). However, it assumes that vortices do not accumulate near the boundary, which is not necessarily true.

Corollary 1. When the assumptions of [31] are satisfied, i.e. $u^{\varepsilon} \in S^{1}\left(u^{\varepsilon}{ }_{3} \equiv 0\right)$ with a lifting $\varphi_{\varepsilon},\left\|\varphi_{\varepsilon}\right\|_{L^{\infty}} \leq N$, then $D_{\varepsilon} \equiv 0$, and we get

$$
\begin{gathered}
p_{\varepsilon} \text { bounded in } B V_{\mathrm{loc}}\left(\mathbb{R}^{+}\right) \quad p_{\varepsilon}-p \text { in } B V_{\mathrm{loc}}\left(\mathbb{R}^{+}\right) \\
\liminf _{\varepsilon \rightarrow 0} E_{\varepsilon}\left(u^{\varepsilon}\right) \geq\|p\|_{B V\left(\mathbb{R}^{+}\right)} \geq\left|\int_{0}^{+\infty} p^{\prime}\right|=|p(0)| \geq|\partial \Omega| .
\end{gathered}
$$

Remark 3.1. This is consistent with the lower bound obtained in [30] which was

$$
\liminf _{\varepsilon \rightarrow 0} E_{\varepsilon}\left(u^{\varepsilon}\right) \geq \int_{\Omega}\left|\operatorname{div}\left(\varphi u+u^{\perp}\right)\right| .
$$

Indeed, this can be proved rather simply. For any $f \in C^{\infty}(\mathbb{R})$ such that $|f| \leq 1$ and $f(0)=0$,

$$
\int_{\Omega} f(g) \operatorname{div}\left(\varphi u+u^{\perp}\right)=-\int_{\Omega} f^{\prime}(g)
$$

(after integrating by parts and using $u=-\nabla^{\perp} g,|\nabla g|=1$ ). Using the coarea formula of Federer,

$$
-\int_{\Omega} f^{\prime}(g)=-\int_{\mathbb{R}} f^{\prime}(t) p(t) \mathrm{d} t .
$$

Taking the supremum over all such $f$, we obtain that

$$
\int_{\Omega}\left|\operatorname{div}\left(\varphi u+u^{\perp}\right)\right| \geq\|p\|_{B V\left(\mathbb{R}^{+}\right)}
$$

In addition, there is equality in (3.10) if and only if $p^{\prime} \leq 0$ on $\mathbb{R}^{+}$(for $g \geq 0$ ), which is to be compared to the sign condition obtained in $[30,31]$.

Remark 3.2. If vortices $a_{i}$ with degrees $\mathrm{d}_{i}$ are fixed a priori, then the lower bound (3.8) of Theorem 3 implies the "nice" formula

$$
\liminf _{\varepsilon \rightarrow 0} E_{\varepsilon}\left(u^{\varepsilon}\right) \geq\left|\int_{\mathbb{R}} p^{\prime}+2 \pi D\right|=|| \partial \Omega\left|-2 \pi \sum_{i} \mathrm{~d}_{i} g\left(a_{i}\right)\right| .
$$


As a corollary of Theorem 3, we can derive a lower bound in the general case when $g$ changes sign. We define $\Omega_{+}$to be the interior of the set $\{g \geq 0\}$ and $\Omega_{-}$the interior of $\{g \leq 0\}$,

$$
p_{+}(t)=\mathcal{H}^{1}(\{g=t\}) \quad \text { for } t>0
$$

extended by the values 0 for $t>\max g,\left|\partial \Omega_{+}\right|$for $t<0$;

$$
p_{-}(t)=\mathcal{H}^{1}(\{g=t\}) \quad \text { for } t<0
$$

extended by 0 for $t<\min g,\left|\partial \Omega_{-}\right|$for $t>0$. For $t \geq 0$,

$$
D_{\varepsilon}^{+}(t)=\frac{1}{2 \pi} \int_{\left\{g_{\varepsilon}=t\right\} \cap \Omega_{+}} V_{\varepsilon} \cdot \tau=\sum_{i / g_{\varepsilon}\left(a_{i}^{\varepsilon}\right) \geq t} \mathrm{~d}_{i}^{\varepsilon} \in \mathbb{Z},
$$

and for $t \leq 0$,

$$
D_{\varepsilon}^{-}(t)=\frac{1}{2 \pi} \int_{\left\{g_{\varepsilon}=t\right\} \cap \Omega_{-}} V_{\varepsilon} \cdot \tau=\sum_{i / g_{\varepsilon}\left(a_{i}^{\varepsilon}\right) \leq t} \mathrm{~d}_{i}^{\varepsilon} \in \mathbb{Z},
$$

and we set

$$
M_{\varepsilon}^{ \pm}=2 \pi \int_{0}^{t} D_{\varepsilon}^{ \pm}(s) \mathrm{d} s
$$

Obviously, the contributions of energy on $\Omega_{+}$and $\Omega_{-}$can be added up and we deduce from Theorem 3 , applied successively on $\Omega_{+}$and $\Omega_{-}$.

Theorem 3'. Assume $E_{\varepsilon}\left(u^{\varepsilon}\right) \leq C$, then as $\varepsilon \rightarrow 0$,

$$
\begin{gathered}
p_{\varepsilon} \rightarrow p_{+} \quad \text { in } L^{1}\left(\mathbb{R}^{+}\right) \\
p_{\varepsilon} \rightarrow p_{-} \text {in } L^{1}\left(\mathbb{R}^{-}\right) \\
p_{\varepsilon}+M_{\varepsilon}^{+} \text {is bounded in } B V_{\mathrm{loc}}\left(\mathbb{R}^{+}\right) \\
p_{\varepsilon}+M_{\varepsilon}^{-} \text {is bounded in } B V_{\mathrm{loc}}\left(\mathbb{R}^{-}\right),
\end{gathered}
$$

there exist $M^{+}, M^{-} \in L^{1}, D^{+}=\frac{\left(M^{+}\right)^{\prime}}{2 \pi}, D_{-}=\frac{\left(M^{-}\right)^{\prime}}{2 \pi}$, such that

$$
\begin{gathered}
p_{\varepsilon}+M_{\varepsilon}^{+} \rightarrow p_{+}+M_{+} \quad \text { in } B V_{\mathrm{loc}}\left(\mathbb{R}^{+}\right) \\
p_{\varepsilon}+M_{\varepsilon}^{-} \rightarrow p_{-}+M_{-} \quad \text { in } B V_{\mathrm{loc}}\left(\mathbb{R}^{-}\right) \\
p_{\varepsilon}^{\prime}+2 \pi D_{\varepsilon}^{+} \rightarrow p_{+}^{\prime}+2 \pi D \text { in } \mathcal{M}\left(\mathbb{R}^{+}\right)(\text {respectively }-)
\end{gathered}
$$

and we have

$$
\begin{aligned}
\liminf _{\varepsilon \rightarrow 0} E_{\varepsilon}\left(u^{\varepsilon}\right) & \geq\left\|p^{+}+M^{+}\right\|_{B V\left(\mathbb{R}^{+}\right)}+\left\|p^{-}+M^{-}\right\|_{B V\left(\mathbb{R}^{-}\right)} \\
& =\int_{0}^{+\infty}\left|\left(p^{+}\right)^{\prime}+2 \pi D^{+}\right|+\int_{-\infty}^{0}\left|\left(p^{-}\right)^{\prime}+2 \pi D^{-}\right| .
\end{aligned}
$$

If we have the extra assumption on a set $\{-\delta \leq g \leq \delta\}$, then

$$
\liminf _{\varepsilon \rightarrow 0} E_{\varepsilon}\left(u^{\varepsilon}\right) \geq\left\|p^{+}+M^{+}\right\|_{B V(\mathbb{R})}+\left\|p^{-}+M^{-}\right\|_{B V(\mathbb{R})} .
$$


We start with the

Proof of Lemma 3.1. First of all, let us show that the weak limit of $p_{\varepsilon}$ is $p$. Let $f \in C^{0}\left(\mathbb{R}^{+}\right)$be a test-function. Using the coarea formula, we have

$$
\int_{\mathbb{R}^{+}} f(t) p_{\varepsilon}(t) \mathrm{d} t=\int_{\mathbb{R}^{+}} f(t)\left(\int_{\left\{g_{\varepsilon}=t\right\} \cap \Omega}\left|\nabla g_{\varepsilon}\right|\right) \mathrm{d} t=\int_{\left\{g_{\varepsilon} \geq 0\right\} \cap \Omega} f\left(g_{\varepsilon}\right)\left|\nabla g_{\varepsilon}\right|^{2} .
$$

But we know that $\left|\nabla g_{\varepsilon}\right| \rightarrow 1$ in $\cap_{p<\infty} L^{p}$, thus

$$
\int_{\mathbb{R}^{+}} f(t) p_{\varepsilon}(t) \mathrm{d} t=\int_{\left\{g_{\varepsilon} \geq 0\right\} \cap \Omega} f\left(g_{\varepsilon}\right)+o(1),
$$

while $g_{\varepsilon} \rightarrow g$ uniformly, hence $f\left(g_{\varepsilon}\right) \mathbf{1}_{g_{\varepsilon} \geq 0} \rightarrow f(g) \mathbf{1}_{\Omega}$ a.e. It follows by dominated convergence that

$$
\int_{\mathbb{R}^{+}} f(t) p_{\varepsilon}(t) \mathrm{d} t \rightarrow \int_{\Omega} f(g) .
$$

On the other hand, using once again the coarea formula,

$$
\int_{\Omega} f(g)=\int_{\Omega} f(g)|\nabla g|=\int_{\mathbb{R}^{+}} f(t) p(t) \mathrm{d} t .
$$

Hence,

$$
\int_{\mathbb{R}^{+}} f(t) p_{\varepsilon}(t) \mathrm{d} t \rightarrow \int_{\mathbb{R}^{+}} f(t) p(t) \mathrm{d} t
$$

Therefore, the weak limit of $p_{\varepsilon}$ is $p$. In order to prove weak $L^{1}$ convergence, we just prove that $p_{\varepsilon}$ is equiintegrable in $\mathbb{R}^{+}$. First of all, $p \in L^{1}\left(\mathbb{R}^{+}\right)$because $\int_{\mathbb{R}^{+}} p(t) \mathrm{d} t=\int_{\Omega} 1=|\Omega|$ as in (3.14). Hence, for $\eta>0$ given, there exists $\delta$ such that

$$
|b-a|<\delta \Longrightarrow \int_{a}^{b} p(t) \mathrm{d} t<\eta
$$

Secondly,

$$
\int_{a}^{b} p_{\varepsilon}(t) \mathrm{d} t=\int_{a \leq g_{\varepsilon} \leq b}\left|\nabla g_{\varepsilon}\right|^{2} \leq\left\|\nabla g_{\varepsilon}\right\|_{L^{4}}^{2} \operatorname{vol}\left(a \leq g_{\varepsilon} \leq b\right)^{\frac{1}{2}} \leq C \operatorname{vol}\left(a \leq g_{\varepsilon} \leq b\right)^{\frac{1}{2}}
$$

By strong convergence of $g_{\varepsilon}$ to $g$, for $\varepsilon$ small enough, and for all $a, b \in \mathbb{R}^{+}$, we have (with the same coarea formula again)

$$
\operatorname{vol}\left(a \leq g_{\varepsilon} \leq b\right) \leq \operatorname{vol}\left(a-\frac{\delta}{4} \leq g \leq b+\frac{\delta}{4}\right)=\int_{a-\frac{\delta}{4} \leq g \leq b+\frac{\delta}{4}}|\nabla g|=\int_{a-\frac{\delta}{4}}^{b+\frac{\delta}{4}} p(t) \mathrm{d} t .
$$

Thus, in view of $(3.15)$, if $|b-a|<\frac{\delta}{2}$, we have

$$
\int_{a}^{b} p_{\varepsilon}(t) \mathrm{d} t \leq C \eta^{\frac{1}{2}}
$$


This characterizes the equiintegrability of $p_{\varepsilon}$, which implies that $p_{\varepsilon} \rightarrow p$ in $L^{1}\left(\mathbb{R}^{+}\right)$.

Proof of Theorem 3. As already mentioned, we are going to use the fact that $E_{\varepsilon}\left(u^{\varepsilon}\right) \geq \int_{\Omega}\left|H_{\varepsilon} \cdot V_{\varepsilon}\right|$ and bound from below $\int_{\Omega}\left|H_{\varepsilon} \cdot V_{\varepsilon}\right|$. We first can write $H_{\varepsilon} \cdot V_{\varepsilon}$ as follows (using $u^{\varepsilon} \mathbf{1}_{\Omega}+H_{\varepsilon}=-\nabla^{\perp} g_{\varepsilon}$ ):

$$
H_{\varepsilon} \cdot V_{\varepsilon}=-\left(u^{\varepsilon}+\nabla^{\perp} g_{\varepsilon}\right) \cdot V_{\varepsilon} \text { in } \Omega .
$$

One can also check that, since $u^{\varepsilon}$ is $S^{1}$-valued and locally equal to $\mathrm{e}^{i \varphi_{\varepsilon}}$, we have

$$
\operatorname{curl} u^{\varepsilon}=u^{\varepsilon} \cdot V_{\varepsilon} \text { in } \Omega \text {, }
$$

and thus, using curl $H_{\varepsilon}=0$,

$$
H_{\varepsilon} \cdot V_{\varepsilon}=-\nabla^{\perp} g_{\varepsilon} \cdot V_{\varepsilon}-\operatorname{curl} u^{\varepsilon}=-\nabla^{\perp} g_{\varepsilon} \cdot V_{\varepsilon}+\operatorname{curl} \nabla^{\perp} g_{\varepsilon} \quad \text { in } \Omega .
$$

This decomposition is the same trick as the one that we used in [30] to obtain the lower bound. Let us now take a test-function $f \in C^{1}\left(\mathbb{R}^{+}\right)$and evaluate

$$
\int_{\Omega} f\left(g_{\varepsilon}\right)\left(-V_{\varepsilon} \cdot \nabla^{\perp} g_{\varepsilon}+\operatorname{curl} \nabla^{\perp} g_{\varepsilon}\right) .
$$

Then, we will use

to obtain the lower bound.

$$
\int_{\Omega}\left|H_{\varepsilon} \cdot V_{\varepsilon}\right| \geq \sup _{|f| \leq 1} \int_{\Omega} f\left(g_{\varepsilon}\right)\left(-V_{\varepsilon} \cdot \nabla^{\perp} g_{\varepsilon}+\operatorname{curl} \nabla^{\perp} g_{\varepsilon}\right)
$$

Let $\delta>0$ be fixed. We recall that we are in the case $g \geq 0$ and $g=0$ on $\partial \Omega$; therefore, by uniform convergence of $g_{\varepsilon}$ to $g$, for $\varepsilon$ small enough, $\left\{g_{\varepsilon} \geq \delta\right\} \subset \Omega$. Using $F$, some primitive of $f$,

$$
\int_{g_{\varepsilon} \geq \delta} f\left(g_{\varepsilon}\right)\left(V_{\varepsilon} \cdot \nabla^{\perp} g_{\varepsilon}-\operatorname{curl} \nabla^{\perp} g_{\varepsilon}\right)=\int_{g_{\varepsilon} \geq \delta} V_{\varepsilon} \cdot \nabla^{\perp} F\left(g_{\varepsilon}\right)-f\left(g_{\varepsilon}\right) \operatorname{curl} \nabla^{\perp} g_{\varepsilon} .
$$

Now we can observe that

$$
\begin{aligned}
V_{\varepsilon} \cdot \nabla^{\perp} F\left(g_{\varepsilon}\right) & =\operatorname{curl}\left(F\left(g_{\varepsilon}\right) V_{\varepsilon}\right)-F\left(g_{\varepsilon}\right) \operatorname{curl} V_{\varepsilon} \\
& =\operatorname{curl}\left(F\left(g_{\varepsilon}\right) V_{\varepsilon}\right)-2 \pi \sum_{i=1}^{n(\varepsilon)} \mathrm{d}_{i}^{\varepsilon} F\left(g_{\varepsilon}\left(a_{i}^{\varepsilon}\right)\right) \delta_{a_{i}^{\varepsilon}}
\end{aligned}
$$

in the sense of distributions, where the $\left(a_{i}^{\varepsilon}, \mathrm{d}_{i}^{\varepsilon}\right)$ are the vortices of $u^{\varepsilon}(c f .(3.1))$. Hence,

$$
\int_{g_{\varepsilon} \geq \delta} V_{\varepsilon} \cdot \nabla^{\perp} F\left(g_{\varepsilon}\right)=\int_{g_{\varepsilon}=\delta} F\left(g_{\varepsilon}\right) V_{\varepsilon} \cdot \tau-2 \pi \sum_{i / g_{\varepsilon}\left(a_{i}^{\varepsilon}\right) \geq \delta} \mathrm{d}_{i}^{\varepsilon} F\left(g_{\varepsilon}\left(a_{i}^{\varepsilon}\right)\right) .
$$

But in view of definition (3.3), this is

$$
2 \pi F(\delta) D_{\varepsilon}(\delta)-2 \pi \sum_{i / g_{\varepsilon}\left(a_{i}^{\varepsilon}\right) \geq \delta} \mathrm{d}_{i}^{\varepsilon} F\left(g_{\varepsilon}\left(a_{i}^{\varepsilon}\right)\right) .
$$

(Without loss of generality, we may assume that $\left\{g_{\varepsilon}=\delta\right\}$ does not contain any vortex.) Meanwhile, we examine the other term. Integrating by parts, we have

$$
\int_{g_{\varepsilon} \geq \delta} f\left(g_{\varepsilon}\right) \operatorname{curl} \nabla^{\perp} g_{\varepsilon}=\int_{g_{\varepsilon}=\delta} f\left(g_{\varepsilon}\right) \frac{\partial g_{\varepsilon}}{\partial n_{\mathrm{ext}}}-\int_{g_{\varepsilon} \geq \delta} f^{\prime}\left(g_{\varepsilon}\right)\left|\nabla g_{\varepsilon}\right|^{2} .
$$


On the level-set $\left\{g_{\varepsilon}=\delta\right\}$, we have $\frac{\partial g_{\varepsilon}}{\partial n_{\text {ext }}}=-\left|\nabla g_{\varepsilon}\right|$, hence

$$
\int_{g_{\varepsilon} \geq \delta} f\left(g_{\varepsilon}\right) \operatorname{curl} \nabla^{\perp} g_{\varepsilon}=-f(\delta) p_{\varepsilon}(\delta)-\int_{g_{\varepsilon} \geq \delta} f^{\prime}\left(g_{\varepsilon}\right)\left|\nabla g_{\varepsilon}\right|^{2} .
$$

Using the coarea formula, the second term is equal to $-\int_{\delta}^{\infty} f^{\prime}(t) p_{\varepsilon}(t) \mathrm{d} t$. Inserting (3.22) and (3.23) into (3.19), we obtain

$$
\begin{aligned}
\int_{g_{\varepsilon} \geq \delta} f\left(g_{\varepsilon}\right)\left(V_{\varepsilon} \cdot \nabla^{\perp} g_{\varepsilon}-\operatorname{curl} \nabla^{\perp} g_{\varepsilon}\right)= & 2 \pi F(\delta) D_{\varepsilon}(\delta) \\
& -2 \pi \sum_{i / g_{\varepsilon}\left(a_{i}^{\varepsilon}\right) \geq \delta} \mathrm{d}_{i}^{\varepsilon} F\left(g_{\varepsilon}\left(a_{i}^{\varepsilon}\right)\right)+f(\delta) p_{\varepsilon}(\delta)+\int_{\delta}^{\infty} f^{\prime}(t) p_{\varepsilon}(t) \mathrm{d} t .
\end{aligned}
$$

Taking $f \equiv 1$ and letting $\delta \rightarrow 0$, one formally obtains the result mentioned in Remark 3.2 from this equation.

We can transform the second term in the sum as follows: noticing that $D_{\varepsilon}(t)=\sum_{g_{\varepsilon}\left(a_{i}^{\varepsilon}\right) \geq t} \mathrm{~d}_{i}^{\varepsilon}$, let us denote by $t_{k}(k=1, \cdots, K)$ the increasing sequence of $t \geq \delta$ such that $\left\{g_{\varepsilon}=t\right\}$ contains a vortex. Then,

$$
\begin{aligned}
2 \pi \sum_{i / g_{\varepsilon}\left(a_{i}^{\varepsilon}\right) \geq \delta} \mathrm{d}_{i}^{\varepsilon} F\left(g_{\varepsilon}\left(a_{i}^{\varepsilon}\right)\right) & =2 \pi \sum_{k=1}^{K}\left(\sum_{i / g_{\varepsilon}\left(a_{i}^{\varepsilon}\right)=t_{k}} \mathrm{~d}_{i}^{\varepsilon}\right) F\left(t_{k}\right) \\
& =2 \pi \sum_{k=1}^{K}\left(D_{\varepsilon}\left(t_{k}\right)-D_{\varepsilon}\left(t_{k+1}\right)\right) F\left(t_{k}\right) .
\end{aligned}
$$

After a standard re-summation process, (3.25) becomes

$$
2 \pi \sum_{i / g_{\varepsilon}\left(a_{i}^{\varepsilon}\right) \geq \delta} \mathrm{d}_{i}^{\varepsilon} F\left(g_{\varepsilon}\left(a_{i}^{\varepsilon}\right)\right)=2 \pi \sum_{k=2}^{K} D_{\varepsilon}\left(t_{k}\right) \int_{t_{k-1}}^{t_{k}} f(s) \mathrm{d} s+2 \pi D_{\varepsilon}\left(t_{1}\right) F\left(t_{1}\right) .
$$

We observe that $D_{\varepsilon}(\delta)=D_{\varepsilon}\left(t_{1}\right)$ and $D_{\varepsilon}$ is equal to $D_{\varepsilon}\left(t_{k}\right)$ on $\left(t_{k-1}, t_{k}\right]$; therefore,

$$
\begin{aligned}
2 \pi F(\delta) D_{\varepsilon}(\delta)-2 \pi \sum_{i / g_{\varepsilon}\left(a_{i}^{\varepsilon}\right) \geq \delta} \mathrm{d}_{i}^{\varepsilon} F\left(g_{\varepsilon}\left(a_{i}^{\varepsilon}\right)\right) & =-2 \pi \int_{t_{1}}^{t_{K}} D_{\varepsilon}(s) f(s) \mathrm{d} s-2 \pi D_{\varepsilon}\left(t_{1}\right)\left(F\left(t_{1}\right)-F(\delta)\right) \\
& =-2 \pi \int_{\delta}^{\infty} D_{\varepsilon}(t) f(t) \mathrm{d} t
\end{aligned}
$$

(with the convention $p_{\varepsilon}=0$ for $t>\max g_{\varepsilon}$ ). Plugging this into (3.24), we conclude that

$$
\begin{aligned}
\int_{g_{\varepsilon} \geq \delta} f\left(g_{\varepsilon}\right)\left(V_{\varepsilon} \cdot \nabla^{\perp} g_{\varepsilon}-\operatorname{curl} \nabla^{\perp} g_{\varepsilon}\right) & =f(\delta) p_{\varepsilon}(\delta)+\int_{\delta}^{\infty} f^{\prime}(t) p_{\varepsilon}(t) \mathrm{d} t-2 \pi \int_{\delta}^{\infty} D_{\varepsilon}(t) f(t) \mathrm{d} t \\
& =-\int_{\delta}^{\infty} f(t)\left(p_{\varepsilon}^{\prime}(t)+2 \pi D_{\varepsilon}(t)\right) \mathrm{d} t .
\end{aligned}
$$

Going back to (3.18), taking the supremum over all $f^{\prime}$ 's such that $|f| \leq 1$, we deduce that $p_{\varepsilon}^{\prime}+2 \pi D_{\varepsilon}$ is bounded in the sense of measures, or that $p_{\varepsilon}+M_{\varepsilon}$ is bounded in $B V([\delta,+\infty))$. Since this is true for all $\delta>0$, we can say it is bounded in $B V_{\text {loc }}\left(\mathbb{R}^{+}\right)$. Moreover, we have

$$
\int_{g_{\varepsilon} \geq \delta}\left|H_{\varepsilon} \cdot V_{\varepsilon}\right| \geq \int_{\delta}^{\infty}\left|p_{\varepsilon}^{\prime}+2 \pi D_{\varepsilon}\right|
$$


and using the lower semi-continuity of the mass,

$$
\liminf _{\varepsilon \rightarrow 0} \int_{g_{\varepsilon} \geq \delta}\left|H_{\varepsilon} \cdot V_{\varepsilon}\right| \geq \int_{\delta}^{\infty}\left|p^{\prime}+2 \pi D\right| .
$$

Since this is true for all $\delta>0$, we conclude that

$$
\liminf _{\varepsilon \rightarrow 0} E_{\varepsilon}\left(u^{\varepsilon}\right) \geq \liminf _{\varepsilon \rightarrow 0} \int_{g_{\varepsilon} \geq \delta}\left|H_{\varepsilon} \cdot V_{\varepsilon}\right| \geq \int_{0}^{\infty}\left|p^{\prime}+2 \pi D\right|,
$$

which provides the desired result.

Next, we consider the particular case in which $u^{\varepsilon}$ does not have vortices in a set of the form $\{g \leq \delta\}$ for some small $\delta$, and thus has a lifting $\varphi_{\varepsilon}$ which is assumed to be uniformly bounded in $L^{\infty}$ in this neighborhood. Since $g_{\varepsilon} \rightarrow g$ uniformly, we have $\cup_{\varepsilon_{k}} \cap_{\varepsilon<\varepsilon_{k}}\left\{g_{\varepsilon}<\delta\right\} \cap \Omega=\{g<\delta\}$, and we may assume that $u^{\varepsilon}$ has no vortex and has the lifting $\varphi_{\varepsilon}$ in the set $\left\{g_{\varepsilon}<\delta\right\}$ for $\varepsilon$ small enough. Then, we are in the framework of [30] and

$$
H_{\varepsilon} \cdot V_{\varepsilon}=H_{\varepsilon} \cdot \varphi_{\varepsilon}=-u^{\varepsilon} \cdot \nabla \varphi_{\varepsilon}-\nabla^{\perp} g_{\varepsilon} \cdot \nabla \varphi_{\varepsilon}=-\operatorname{div}\left(\varphi_{\varepsilon} \nabla^{\perp} g_{\varepsilon}\right)-\operatorname{curl} u^{\varepsilon}=\operatorname{div}\left(-\varphi_{\varepsilon} \nabla^{\perp} g_{\varepsilon}+u^{\varepsilon \perp}\right) .
$$

Then,

$$
\begin{aligned}
C \geq \int_{\left\{g_{\varepsilon}<\delta\right\} \cap \Omega}\left|H_{\varepsilon} \cdot V_{\varepsilon}\right| & =\int_{\left\{g_{\varepsilon}<\delta\right\} \cap \Omega}\left|\operatorname{div}\left(-\varphi_{\varepsilon} \nabla^{\perp} g_{\varepsilon}+u^{\varepsilon \perp}\right)\right| \\
& \geq \int_{\cup_{\varepsilon_{k}} \cap_{\varepsilon<\varepsilon_{k}}\left\{g_{\varepsilon}<\delta\right\} \cap \Omega=\{g<\delta\}}\left|\operatorname{div}\left(-\varphi_{\varepsilon} \nabla^{\perp} g_{\varepsilon}+u^{\varepsilon \perp}\right)\right| .
\end{aligned}
$$

$\varphi_{\varepsilon}$ being bounded in $L^{\infty}$, it converges weakly in $\cap_{p} L^{p}$ to some $\varphi$; on the other hand $-\nabla^{\perp} g_{\varepsilon} \rightarrow-\nabla^{\perp} g=u$ strongly in $\cap_{p<\infty} L^{p}$ (see Lem. 2.1), thus div $\left(-\varphi_{\varepsilon} \nabla^{\perp} g_{\varepsilon}+u^{\varepsilon \perp}\right)\left\lfloor\{g<\delta\}\right.$ which remains bounded in $L^{1}$ converges weakly to $\operatorname{div}\left(\varphi u+u^{\perp}\right)$, and

$$
\liminf _{\varepsilon \rightarrow 0} \int_{g_{\varepsilon}<\delta}\left|H_{\varepsilon} \cdot V_{\varepsilon}\right| \geq \int_{g<\delta}\left|\operatorname{div}\left(\varphi u+u^{\perp}\right)\right| .
$$

Following exactly the proof of [30] Lemma 5.2, we obtain that

$$
\int_{g<\delta}\left|\operatorname{div}\left(\varphi u+u^{\perp}\right)\right| \geq|p(\delta)-| \partial \Omega||,
$$

(which may not go to 0 as $\delta \rightarrow 0$ ). Then,

$$
E_{\varepsilon}\left(u^{\varepsilon}\right) \geq \int_{g_{\varepsilon}<\delta}\left|H_{\varepsilon} \cdot V_{\varepsilon}\right|+\int_{g_{\varepsilon} \geq \delta}\left|H_{\varepsilon} \cdot V_{\varepsilon}\right|
$$

and using (3.28) together with (3.31) and (3.32), we are led to

$$
\liminf _{\varepsilon \rightarrow 0} E_{\varepsilon}\left(u^{\varepsilon}\right) \geq \int_{\delta}^{+\infty}\left|p^{\prime}+2 \pi D\right|+|p(\delta)-| \partial \Omega|| .
$$

This is true for all $\delta>0$, hence it implies that

$$
\liminf _{\varepsilon \rightarrow 0} E_{\varepsilon}\left(u^{\varepsilon}\right) \geq \int_{-\infty}^{+\infty}\left|p^{\prime}+2 \pi D\right|
$$


(in the sense of the $B V$ norm of $p+M$ ), where $p$ has been extended by $|\partial \Omega|$ for $t<0$ and $D$ by 0 for $t<0$. This concludes the proof of Theorem 3, from which Theorem 3' also follows.

Remark 3.3. The lower bounds of Theorems 3 and 3' are not always optimal. To improve them, one should first sum up the contributions of $p_{j}^{\prime}+2 \pi D_{j}$ over all the connected components $\mathcal{C}_{j}$ of $g_{\varepsilon}$. We also believe that an optimal bound should involve $\int_{\mathbb{R}^{2}}\left|p^{\prime}+2 \pi \hat{D}\right|+2 \pi|D-\hat{D}|$ where $\hat{D}$ is the truncation of $D: \hat{D}=D$ if $|D| \leq 1$, $\hat{D}=\operatorname{sign}(D)$ otherwise. Thus a total degree one on the boundary of each connected component of $g_{\varepsilon}$ should be favored.

\section{EXAMPLES AND OPTIMAL WALL JUMPS}

In this section, we would like to explain in several examples the different formulas obtained before. We begin with very simple situations in which the theory is easily explained, and afterwards, we try to consider more and more complex situations in order to get an intuition about the characteristics of the minimizers. Each time, we try to compare the different formulas and sometimes give the main differences between this situation and the one studied in $[30,31]$. We also show how the optimal energy can be reached using the so-called "cross-tie" walls or Néel walls depending on the magnitude of the jump across the wall.

We start with the lower bound of Section 2 (for $u \in B V(\Omega)$ )

$$
E(u)=\int_{\Sigma_{u}} A(X) \mathrm{d} \mathcal{H}^{1}
$$

where $X$ was defined in (1.14) and $A$ is given by

$$
\begin{aligned}
& A(X)=2|\sin X-X \cos X| \text { for } X \in\left[0, \frac{\pi}{4}\right] \\
& A(X)=2\left|\left(X-\frac{\pi}{2}\right) \cos X-\sin X+\sqrt{2}\right| \text { for } X \in\left[\frac{\pi}{4}, \frac{\pi}{2}\right] .
\end{aligned}
$$

We will show that this formula is optimal, and that such bounds are achieved for Néel walls in the first case, and "cross-tie" walls in the second case.

We remark that this formula does not say anything about the possible convergence of the vortices and optimal places where they should be put. To see that, we can use the following two formulas for the limiting energy ( $c f .(1.31)$ and (1.32)) only valid if the vortices are of finite number and converge to the points $a_{i}$ with degree $\mathrm{d}_{i}$.

$$
E_{0}\left(u,\left\{a_{i}\right\},\left\{d_{i}\right\}\right)=|\partial \Omega|-2 \pi \sum_{i} \mathrm{~d}_{i} g\left(a_{i}\right)
$$

which may be useful if we know the function $g$ (e.g. it is the distance to the boundary), or equivalently the formula using the perimeter of the level-sets of $g$

$$
E_{0}(u, D)=\int\left|p^{\prime}(t)+2 \pi D(t)\right| \mathrm{d} t .
$$

In particular, if we know that the configuration we are looking at is approximated by a sequence $\left(u^{\varepsilon}\right)$ for which the number of vortices is bounded, then $u^{\varepsilon}$ can be considered as purely planar away from these points and following the arguments of [30], we get as a limiting energy

$$
E_{0}(u)=\int_{\Sigma_{u}} 2|\sin X-X \cos X| \mathrm{d} \mathcal{H}^{1} .
$$


In other words, if we want to reach the optimal wall energy for big jumps given by the second formula (4.3), which is smaller than (4.6), we need to consider sequences with unbounded number of vortices.

\subsection{Limiting energy on simple configurations}

\subsubsection{The unit disc}

Let us begin with the simplest one vortex situation. Namely, we consider the case where $\Omega$ is the unit disc and the limiting (divergence free) vectorfield $u_{0}$ is given by

$$
u_{0}=-\nabla^{\perp} g
$$

where $g(x)=\operatorname{dist}(x, \partial \Omega)$. As already explained in the introduction, it is possible to find a sequence $\left(u^{\varepsilon}\right)$ such that

$$
\begin{aligned}
& u^{\varepsilon} \in H^{1}\left(\Omega, S^{2}\right), \\
& u^{\varepsilon} \longrightarrow u_{0} \text { in } L^{2} \text { strong as } \varepsilon \longrightarrow 0, \\
& E_{\varepsilon}\left(u^{\varepsilon}\right) \longrightarrow 0 \text { as } \varepsilon \longrightarrow 0 .
\end{aligned}
$$

This case is clearly already very different from [30] where the energy is always bounded from below by $|\partial \Omega|$.

However, since $u_{0}$ possesses no line singularity, the formula (4.1) gives the correct lower bound for the energy since the singular set has no $\mathcal{H}^{1}$ measure and thus the integration reduces to zero. In [24], it is proved that this is the only case where a zero limiting energy can be achieved. Moreover, it is now possible to get different lower bounds according to the way the vortices converge. Indeed, imagine that $u^{\varepsilon}$ possesses a degree one vortex that converges to some point $P$ inside the disc. Then, applying the formula (4.4), we get the lower bound

$$
\begin{aligned}
\liminf _{\varepsilon \rightarrow 0} E_{\varepsilon}\left(u^{\varepsilon}\right) & \geq|\partial \Omega|-2 \pi g(P), \\
& =2 \pi \operatorname{dist}(O, P),
\end{aligned}
$$

where $O$ stands for the center of the disc.

This amount of remaining energy comes from the fact that in order for $\left(u^{\varepsilon}\right)$ to converge to $u_{0}$, a concentration of energy must occur on a line joining $P$ to $O$. Along this line, the vectorfield possesses a phase jump of $2 \pi$ which costs at least the energy given above.

An explicit computation of the lower bound is also possible using the formula (4.5). Indeed, for a value $t \in(0,1]$, the level set $g^{-1}(t)$ is precisely the circle of center $O$ and radius $t$ whose perimeter is

$$
p(t)=2 \pi(1-t) .
$$

Now, we must compute the degree of these level sets. It is clear that if $P$ is inside the level set $g^{-1}(t)$ then the degree $D(t)$ will be equal to one. Otherwise the degree will be equal to zero.

Finally, $P$ is inside $g^{-1}(t)$ for all $t \in[0, g(P))$ and outside for all $t \in(g(P), 1]$. Putting all together in the formula, we get the lower bound

$$
\liminf _{\varepsilon \rightarrow 0} E_{\varepsilon}\left(u^{\varepsilon}\right) \geq \int_{0}^{1}\left|p^{\prime}(t)+2 \pi D(t)\right| \mathrm{d} t,=\int_{0}^{g(P)} 0 d t+\int_{g(P)}^{1} 2 \pi \mathrm{d} t,=2 \pi(1-g(P)),
$$

which is the correct answer.

\subsubsection{The unit square}

Consider now the case where $\Omega$ is the unit square of $\mathbb{R}^{2}$, and $u_{0}$ is still given by

$$
u_{0}=-\nabla^{\perp} g,
$$




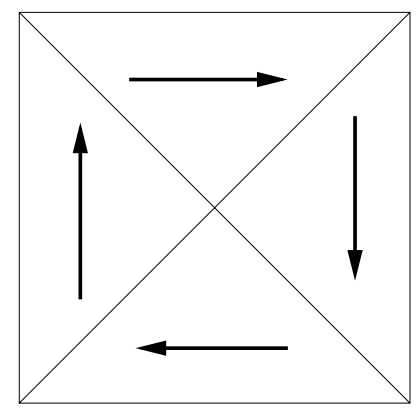

Figure 2. The unit square viscosity solution.

where $g(x)=\operatorname{dist}(x, \partial \Omega)$. Figure 2 shows the vectorfield $u_{0}$. Since $u_{0}$ possesses line singularities, we expect the lower bound of the energy to be strictly positive. Let us use the formula (4.1) to compute the value of the smallest lower bound. On each diagonal segment, $u_{0}$ has a jump of $\frac{\pi}{2}$ in the phase, so that the energy is computable by

$$
\begin{aligned}
\liminf _{\varepsilon \rightarrow 0} E_{\varepsilon}\left(u^{\varepsilon}\right) & \geq \frac{8 \sqrt{2}}{2}\left|\sin \left(\frac{\pi}{4}\right)-\frac{\pi}{4} \cos \left(\frac{\pi}{4}\right)\right| \\
& =4-\pi \\
& >0 .
\end{aligned}
$$

Using the formula (4.4) and assuming that the sequence $\left(u^{\varepsilon}\right)$ possesses a degree one vortex that converges to some point $P$ inside $\Omega$, we get

$$
\begin{aligned}
\liminf _{\varepsilon \rightarrow 0} E_{\varepsilon}\left(u^{\varepsilon}\right) & \geq|| \partial \Omega|-2 \pi g(P)| \\
& =(4-2 \pi \operatorname{dist}(P, \partial \Omega))
\end{aligned}
$$

In the case where $P$ is equal to the center of the square, we of course recover the preceding result $(\operatorname{dist}(P, \partial \Omega)$ $\left.=\frac{1}{2}\right)$. Using the formula (4.5) yields the same answer, and we leave the reader doing the computation.

\subsubsection{The $2 \times 1$ rectangle}

This geometry is quite famous in the micromagnetic community since it has been used as a benchmark for computing rectangular thin films. A recent paper by Rave and Hubert [29] is for instance devoted to the systematic comparison of different configurations in terms of energy, depending on the size and the thickness of the film.

Here, we would like to compare from the point of view of our theory the two usually called Landau-Lifshitz and diamond configurations (see Fig. 3).

For the diamond configuration, there is essentially no problem since all the jumps involved are of magnitude less than $\pi / 2$. It is thus easily seen that in that case, we must use the first formula (4.2). We can also remark that the diamond configuration is only two squares glued together (see Fig. 4) from which we deduce that the optimal achievable energy is twice that of one square

$$
E_{\text {diamond }}=2(4-\pi)=8-2 \pi \text {. }
$$

Moreover, this energy is achievable if the approximate configurations possess two vortices converging to the center of each square. 

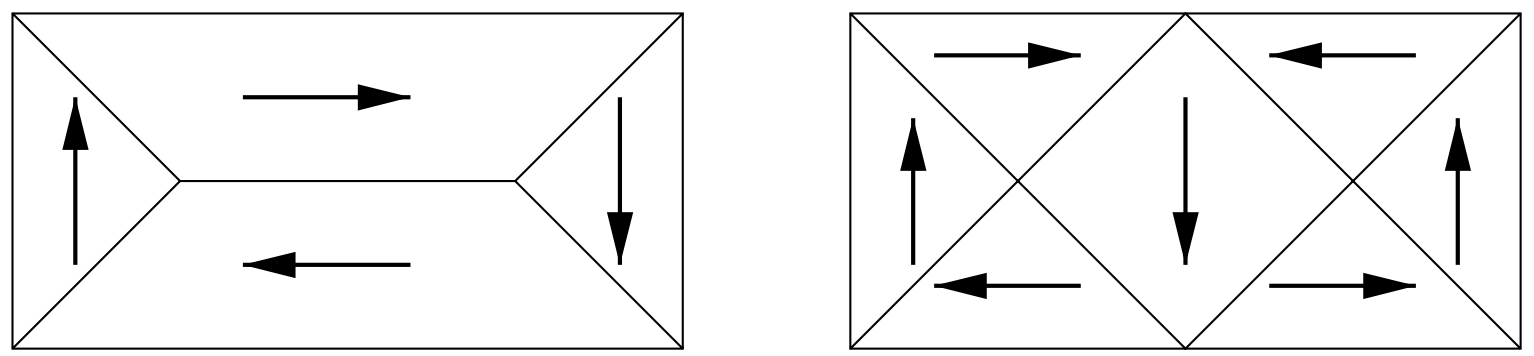

FiguRE 3. Landau Lifshitz and diamond configurations on a rectangle.

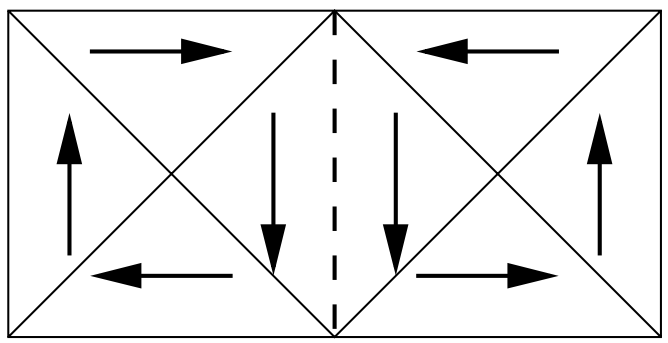

Figure 4. Rectangle as two glued squares.

For the Landau-Lifshitz configuration, the situation is slightly more complicated. In order to compute its energy, we must first look at the way the sequence $\left(u^{\varepsilon}\right)$ converges to this state. Indeed, in the case the number of vortices is bounded, or there are no vortices at all, thus, equation (4.6) applies and we are led to

$$
E_{L L}=4-\pi+2
$$

(the first term is the energy concentrated along the four diagonal and the last one is the energy along the horizontal line). A too quick conclusion could lead to the misleading inequality

$$
E_{L L}>E_{\text {diamond }}
$$

However, using the formula (4.3) gives the better lower bound

$$
E_{L L}^{\prime}=4-\pi+2(\sqrt{2}-1)
$$

and a numerical evaluation leads to

$$
E_{L L}^{\prime}<E_{\text {diamond }}<E_{L L}
$$

We will see in the next section that $E_{L L}^{\prime}$ (which cannot be achieved with a bounded number of votices) corresponds to putting a cross-tie wall in the horizontal line, whereas the first computation of the energy $\left(E_{L L}\right)$ corresponds to putting a Néel wall in this line. This is exactly the kind of behavior that was found in [29] (Fig. 5) where the cross-tie configuration possesses an energy very close to but smaller than the one of the diamond configuration and the Landau-Lifshitz configuration without a cross-tie wall has a much bigger energy.

\subsection{Optimal walls}

To conclude this section, we show that the lower bounds given in the previous sections are indeed optimal. The nice phenomenon that occurs is that the energy density given in (4.2) is achieved by a Néel wall (this was already known from the previous work [30]), which is a one-dimensional structure in the wall, but only for 


\section{Wall}

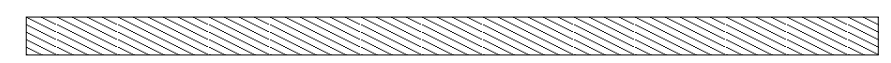

Figure 5. The up-down wall.

jumps in the phase less than $\pi / 2$. For bigger jumps, the Néel wall is no longer optimal and the energy given by (4.3) is achieved with a two-dimensional structure, the "cross-tie walls". As already mentioned, they are actually seen in thin films experiments [21,28]. They are also under investigation by mathematicians [17]. Here, we would like to give a cross-tie wall configuration which is optimal for our model. We begin with the up-down jump where the situation is somewhat simpler, and generalize afterwards to the case of any jump.

\subsubsection{The up-down situation}

In the up-down jump, we have to describe a microscopic structure inside a wall like the one drawn in Figure 5.

We propose the following construction drawn in Figure 6. We decompose the domain into several strips perpendicular to the wall. In each strip, the configuration will be the same, so that the whole configuration is periodic. In the upper half of each strip, we split the domain into three parts (see Fig. 7): the lower left triangle $\Omega_{L}$, the lower right triangle $\Omega_{R}$ and the rest of the domain $\Omega$ (the angles being $\frac{\pi}{4}$ ). We also call $P$ the point on the wall in the middle of the strip. Then, the configuration is given by (see Fig. 7)

$$
u(x)=\left\{\begin{array}{l}
\mathrm{e}^{i \frac{\pi}{4}} \text { on } \Omega_{L}, \\
\mathrm{e}^{-i \frac{\pi}{4}} \text { on } \Omega_{R}, \\
-\nabla^{\perp} \operatorname{dist}(x, P) \text { on } \Omega .
\end{array}\right.
$$

On the lower part of the strip, the configuration is simply extended by symmetry around $P$. In the figures, the circles denote the positions of the vortices at the $\varepsilon$-stage.

Now, we compute the energy of this configuration in order to verify that it is equal to $2(\sqrt{2}-1)$ per unit length of the wall (this is what formula (4.3) gives for an angle of jump equal to $\pi$ ). Since the configuration is periodic, we only need to compute the energy concentrated on the vertical and horizontal lines in one strip assuming the width of the strip is equal to one. As all the jumps are of angle less than $\pi / 2$, they may be achieved by the 1D planar profiles of [30]. We thus compute the energy using the formula of [30] for the energy density

$$
A(X)=2|\sin X-X \cos X|
$$

where $X$ is half the jump in the phase across the wall. To do so, we parameterize the vertical discontinuity by $s$. Since the distance between two neighbouring vortices is assumed to be one, we easily get the half-angle jump $X$ across the vertical line:

$$
X(s)=\left\{\begin{array}{l}
\frac{\pi}{4} \text { if } s \in\left[-\frac{1}{2}, \frac{1}{2}\right] \\
\left|\arctan \left(\frac{1}{2 s}\right)\right| \text { otherwise. }
\end{array}\right.
$$




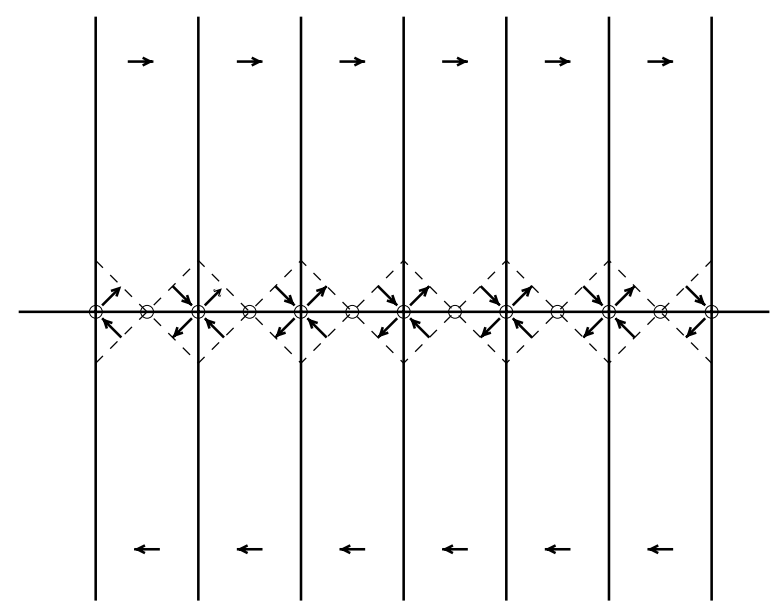

Figure 6. The optimal cross-tie wall.
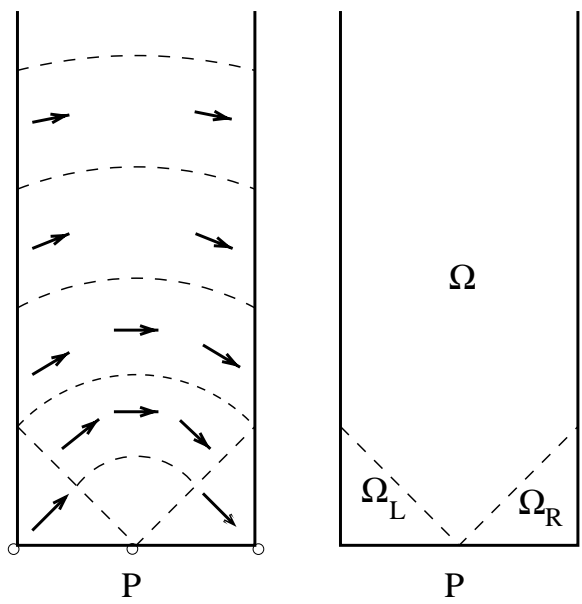

FiguRE 7. Zoom of the upper half strip.

On the other hand, the half-angle jump on the horizontal line jump is constant and equal to $\pi / 4$. Putting everything together gives an energy equal to

$$
\begin{aligned}
E & =4\left(\frac{\sqrt{2}}{2}-\frac{\pi}{4} \frac{\sqrt{2}}{2}\right)+2 \int_{\frac{1}{2}}^{+\infty} 2(\sin (X(s))-X(s) \cos (X(s))) \mathrm{d} s \\
& =\frac{\sqrt{2}}{2}(4-\pi)+4 \int_{\frac{1}{2}}^{+\infty}\left(\frac{1}{\sqrt{1+4 s^{2}}}-\arctan \left(\frac{1}{2 s}\right) \frac{2 s}{\sqrt{1+4 s^{2}}}\right) \mathrm{d} s \\
& =\frac{\sqrt{2}}{2}(4-\pi)+2\left[-\arctan \left(\frac{1}{2 s}\right) \sqrt{1+4 s^{2}}\right]_{\frac{1}{2}}^{+\infty} \\
& =\frac{\sqrt{2}}{2}(4-\pi)+2(\arctan (1) \sqrt{2}-1) \\
& =2(\sqrt{2}-1) .
\end{aligned}
$$



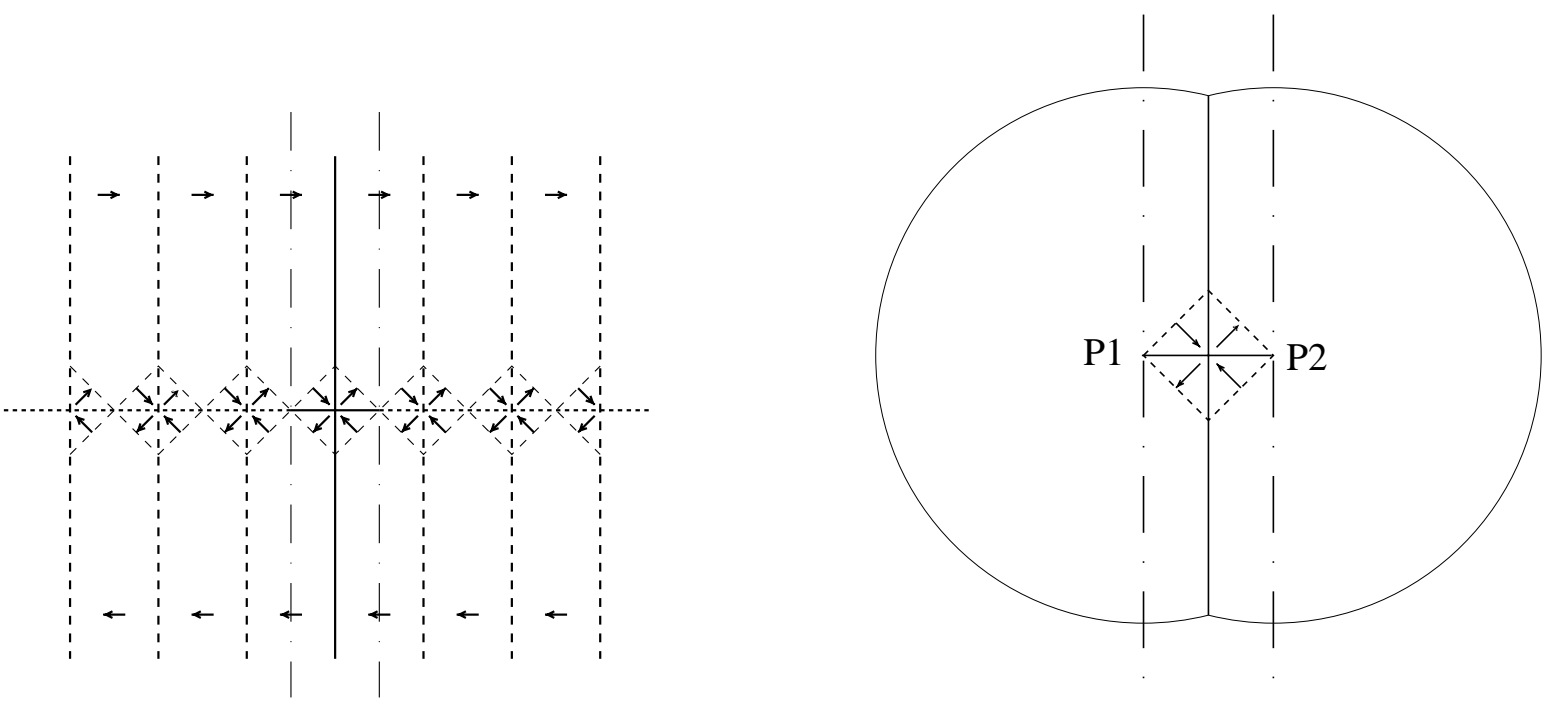

FiguRE 8. Extraction of one strip from the cross-tie pattern.

We also remark that it is possible to do the computation in a more geometrical way. Indeed, we first truncate the domain by two discs centered in $P_{1}$ and $P_{2}$ and of radius $R$ as in Figure 8 and then observe that extending in these two regions the configuration by

$$
u(x)=\left\{\begin{array}{l}
-\nabla^{\perp} \operatorname{dist}\left(x, P_{1}\right) \text { in the left-hand disc, } \\
-\nabla^{\perp} \operatorname{dist}\left(x, P_{2}\right) \text { in the right-hand disc, }
\end{array}\right.
$$

it does not change the total energy since no discontinuity line has been added.

In order to further simplify the computation, we try to recognize $u$ as the orthogonal gradient of the distance function to the boundary of a domain on each side of the vertical discontinuity line. This is not very difficult and a few attempts lead to the egg-like domain drawn in Figure 9. The very last remark before the computation is that one may exchange the left-hand side of the egg-like domain with the reflection of its right-hand side. More precisely, we have to compute the energy of a configuration obtained by gluing two right-hand parts of the egg-like domain. If we decide to glue two right-hand parts (and obtain the diamond-like domain presented in Fig. 9), the configuration obtained by taking the orthogonal gradient of the distance function to the boundary of this new domain possesses exactly the same energy as the original one since the jumps are exactly of the same amount and precisely occur in the same place.

Eventually we observe that now, the domain is convex and the potential is simply the distance function to the boundary. Therefore, we are allowed to use formula (4.4) to compute its energy or at least a lower bound. This leads to the value

$$
E=|\partial \Omega|-2 \pi r
$$

where $r$ is the radius of the biggest disc inscribed in the domain. Recording that $\operatorname{dist}\left(P_{1}, P_{2}\right)=1$, calling $\theta$ the angle $\widehat{P_{2} P_{1} M}$ (see Fig. 9) and $R=\frac{1}{2 \cos (\theta)}$, we get for the perimeter

$$
|\partial \Omega|=4\left(R\left(\theta-\frac{\pi}{4}\right)+\frac{1}{\sqrt{2}}+\frac{\pi}{4}\left(R-\frac{1}{\sqrt{2}}\right)\right) .
$$



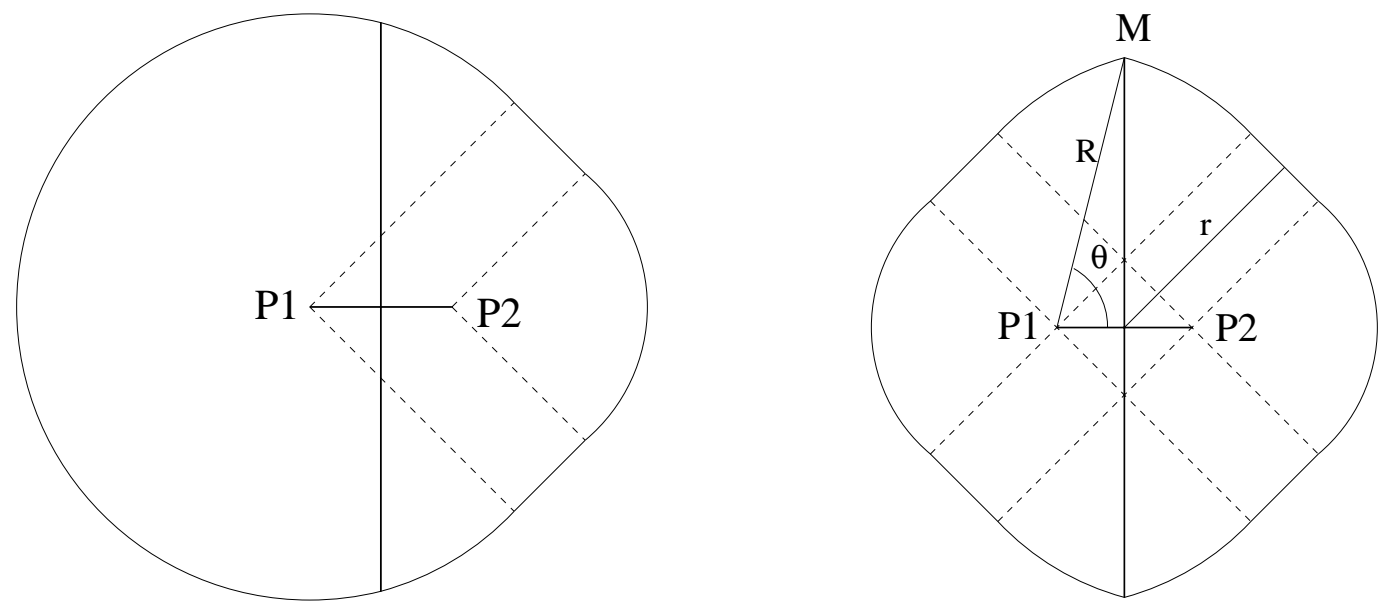

Figure 9. Egg-like domain and diamond-like domain.

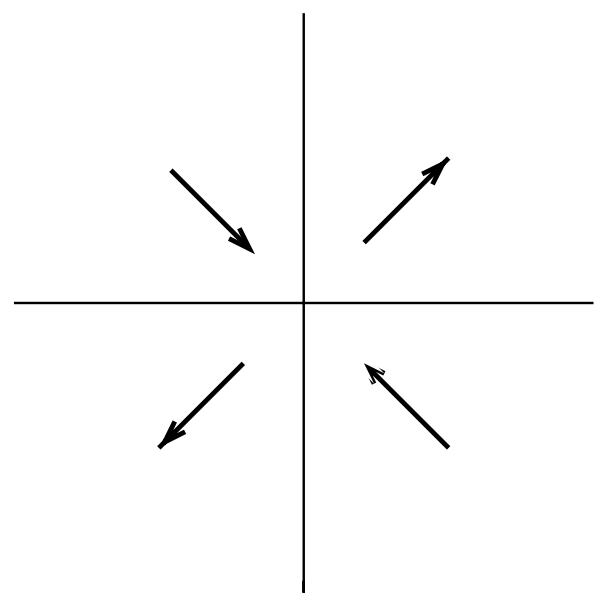

Figure 10. Around the saddle point the configuration consists in 4 domains with constant magnetization.

On the other hand, the biggest inscribed disc has a radius (see Fig. 9)

$$
r=R-\frac{1}{2 \sqrt{2}}
$$

Therefore, the energy of the configuration is given by

$$
\begin{aligned}
E & =|\partial \Omega|-2 \pi r \\
& =4\left(R \theta+\frac{1}{\sqrt{2}}-\frac{\pi}{4 \sqrt{2}}\right)-2 \pi R+\frac{\pi}{\sqrt{2}}, \\
& =2 \sqrt{2}+\frac{2 \theta-\pi}{2 \cos (\theta)}
\end{aligned}
$$




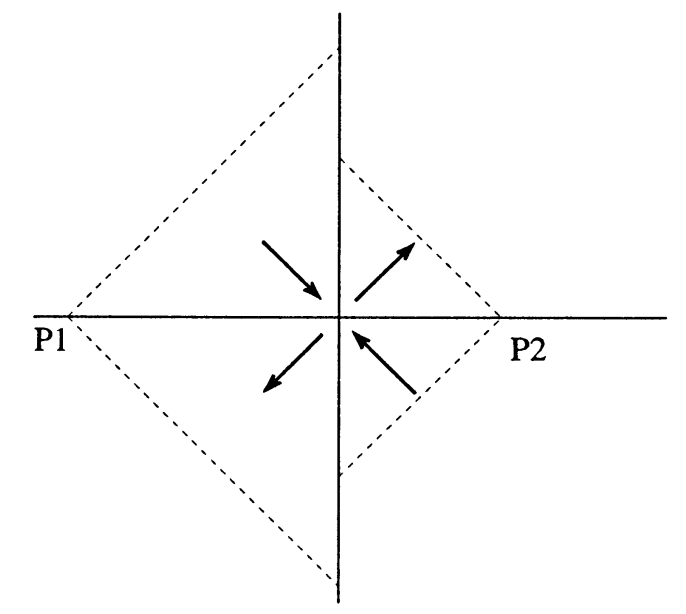

FiguRE 11. triangles with different sizes.

Now letting $\theta$ tend to $\frac{\pi}{2}$ gives

$$
\lim _{\theta \rightarrow \frac{\pi}{2}} E(\theta)=2(\sqrt{2}-1)
$$

which is the desired result.

The computation of this energy is also possible via the level-set theory described in the previous section in a slightly more formal way. Indeed, if we compute the level-sets of the potential $g$ associated to $u$, we find for their degrees

and for their lengths

$$
D(t)=\left\{\begin{array}{l}
-1 \text { if } t \in\left[0, \frac{\sqrt{2}}{4}\right) \\
0 \text { if } t \in\left(\frac{\sqrt{2}}{4},+\infty\right)
\end{array}\right.
$$

The lower bound is now easily computable by

$$
p(t)=\left\{\begin{array}{l}
(4+\pi) t \text { if } t \in\left[0, \frac{\sqrt{2}}{4}\right), \\
\text { a non increasing function of } t \text { if } t \in\left(\frac{\sqrt{2}}{4},+\infty\right) .
\end{array}\right.
$$

$$
\begin{aligned}
E & =\int_{0}^{+\infty}\left|p^{\prime}(t)+2 \pi D(t)\right| \mathrm{d} t \\
& =\int_{0}^{\frac{\sqrt{2}}{4}}|4+\pi-2 \pi| \mathrm{d} t+p\left(\frac{\sqrt{2}}{4}\right)-p(+\infty), \\
& =2 \sqrt{2}-2 .
\end{aligned}
$$

This computation is quite formal since everything has been restricted to only one strip but it is easy to see that doing the computation on the extended configuration drawn in Figure 8 leads exactly to the same result.

Eventually, we realize that making a change of scale of this cross-tie configuration doesn't change its energy since the energy behaves like a length. It is therefore possible to draw a microstructure cross-tie wall which possesses the same energy per unit length. 


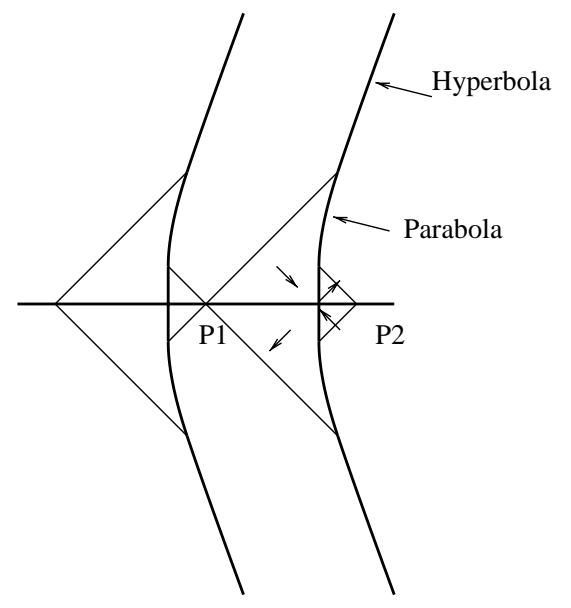

Figure 12. Oblique cross-tie wall (zoom).

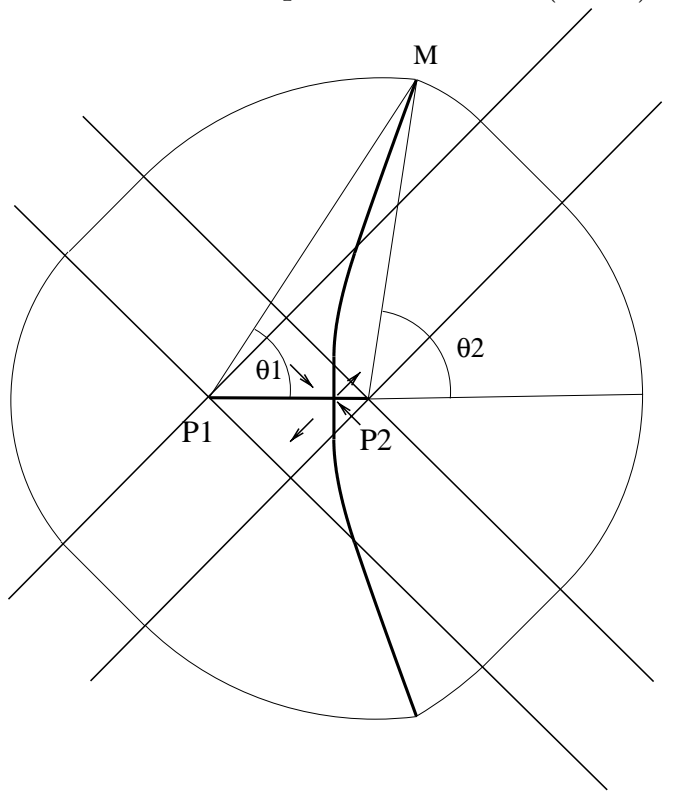

FIGURE 13. Egg-like domain in the oblique case.

4.2.2. The situation with a jump in the phase less than $\pi$

The optimal cross-tie configuration is much more delicate to understand in this situation. Indeed, we must find a configuration where all the angle jumps are less than $\pi / 2$, otherwise these jumps could be replaced by other cross-tie patterns in another smaller scale. Keeping that in mind, we propose the following picture. Around the saddle points we take the same configuration as before (any change here would lead to a bad angle according to the preceding remark). The only remaining freedom is the relative size of these constantly magnetized subdomains. It is possible to consider triangular domains as in Figure 11 and try to extend the configuration outside. On the right hand side of the domain, the solution is taken to be

$$
u(x)=-\nabla^{\perp} \operatorname{dist}\left(x, P_{2}\right),
$$




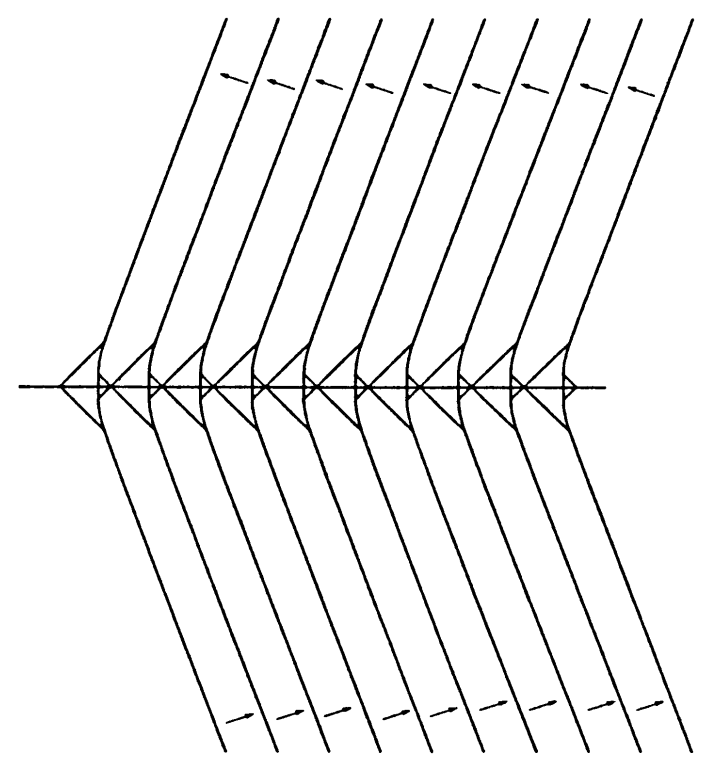

Figure 14. Oblique cross-tie wall.

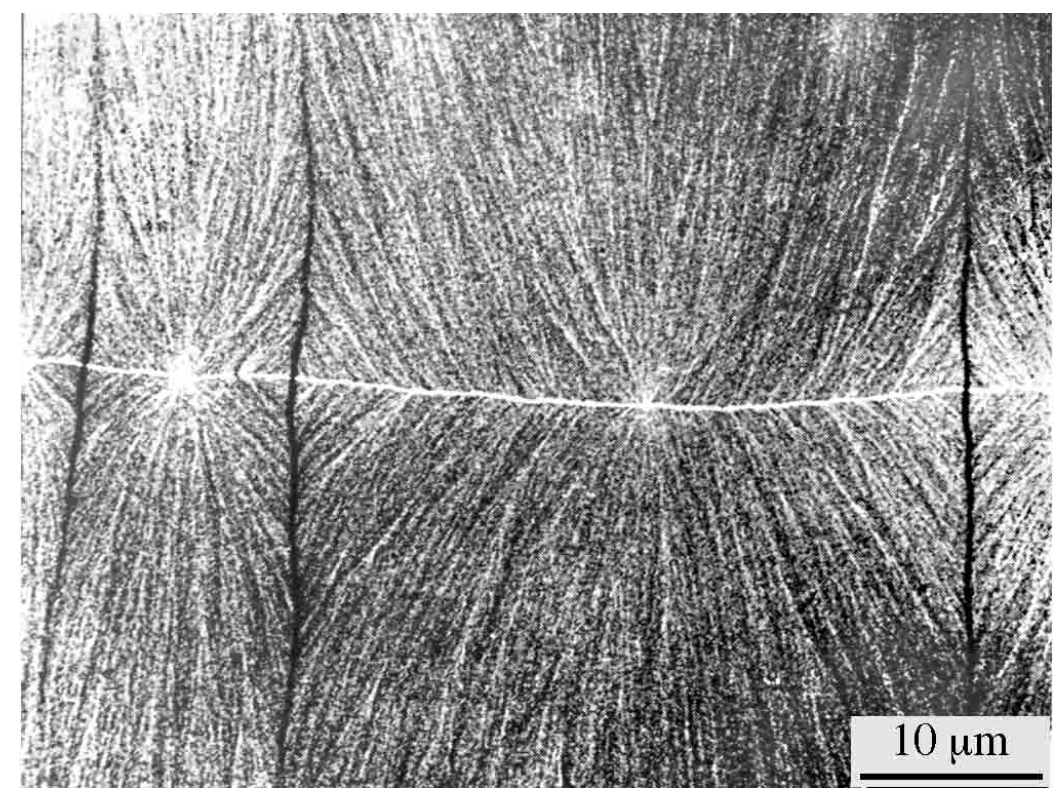

Figure 15. A cross-tie wall in a Permalloy sample (courtesy R. Schäfer).

and we conversely take on the left hand side

$$
u(x)=-\nabla^{\perp} \operatorname{dist}\left(x, P_{1}\right)
$$

Now the problem becomes to find the two curves separating these two solutions in a way which is consistent with the fact that $u$ is divergence-free. An analytical computation of these curves is possible in the same spirit as when one finds the shock curve of a weak solution of a hyperbolic scalar partial differential equation from 
a)

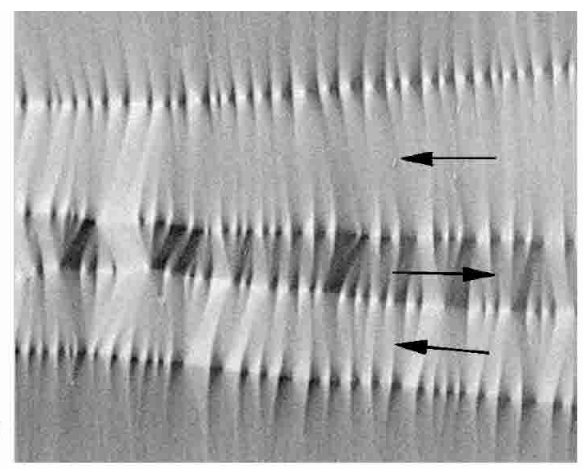

c)

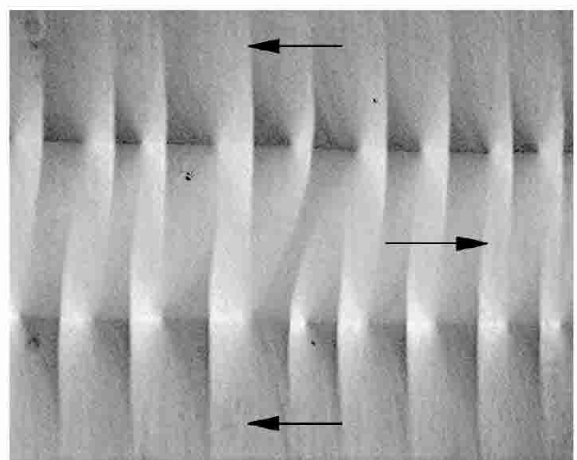

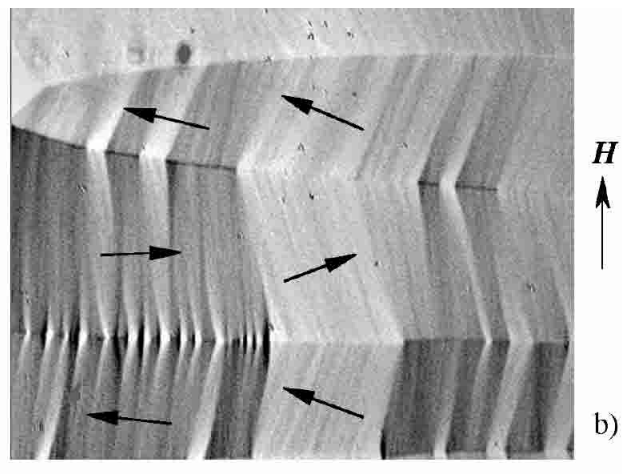

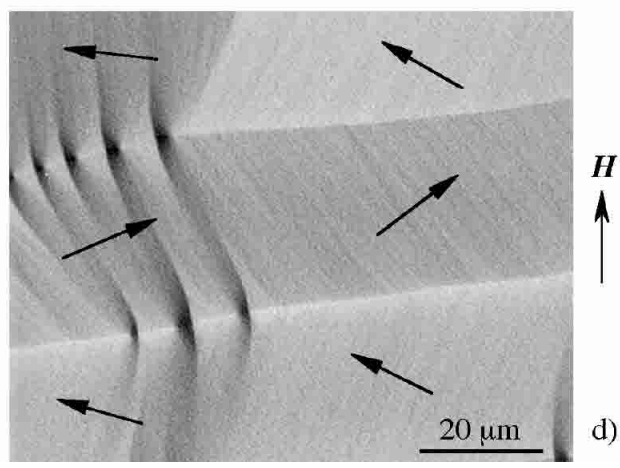

FIgURE 16. Oblique cross-tie walls in Permalloy (courtesy R. Schäfer).

Rankine-Hugoniot relation. However, a very simple argument shows that, in our case, this discontinuity line consists of a piece of parabola and a piece of hyperbola as in Figure 12. Indeed, the first part of this curve separates two regions where the characteristic lines are straight lines of direction $\pi / 4$ on the left hand side and straight lines emerging from $P_{2}$ on the right hand side. Noticing that the tangent to the discontinuity line bisects the angle made by the characteristic on both sides, we get that this discontinuity curve exactly transforms rays emerging from $P_{2}$ into rays of direction $\pi / 4$ by Fermat's reflection principle. It is therefore a parabola of focal point $P_{2}$ and direction $\pi / 4$. Farther, the curve transforms light rays emerging from $P_{2}$ into light rays emerging from $P_{1}$ and is therefore a hyperbola of focal points $P_{1}$ and $P_{2}$.

In order to compute the energy of this configuration, we need to do as in the previous case an integration along the jump lines. Such a computation is possible but really tedious. Thus, we just present a justification of the formula relying on the geometric point of view by finding a convex domain in which the orthogonal gradient of the distance to the boundary function possesses the same jumps as $u$, and using the formula

$$
E(u)=|\partial \Omega|-2 \pi r,
$$

where $r$ is the radius of the biggest disc inscribed in $\Omega$ to obtain a lower bound of the energy. This lower bound should, as in the straight case, be optimal and give the same result as the integration of the wall energy along the discontinuity line. (To really prove this one needs to check that we have equalities in $(1.22,1.23)$ for this distance function.)

We begin by cutting one strip of the pattern and close it by extending the solution to two discs. One centered in $P_{1}$ and of radius $R+C$ and the other centered in $P_{2}$ and of radius $R$. The constant $C$ is tuned in order that both circles intersect at the hyperbola. In other words, the equation of the hyperbola is

$$
\operatorname{dist}\left(x, P_{1}\right)=\operatorname{dist}\left(x, P_{2}\right)+C .
$$


As before, interchanging the right hand parts of the egg-like domains with the left-hand parts leads to a domain drawn in Figure 13, which is still convex and on which we have to compute the energy of the orthogonal gradient of the distance to the boundary function.

We now use the formula (4.9). In view of the shape of the domain (see Fig. 13 for notations), we get for the left hand part of the boundary

$$
\left|(\partial \Omega)_{L}\right|=2\left(\left(\frac{3 \pi}{4}-\theta_{2}\right) R+\frac{1}{\sqrt{2}}+\frac{\pi}{4}\left(R-\frac{1}{\sqrt{2}}\right)\right),
$$

and for the right hand part

$$
\left|(\partial \Omega)_{R}\right|=2\left(\left(\theta_{1}-\frac{\pi}{4}\right)(R+C)+\frac{1}{\sqrt{2}}+\frac{\pi}{4}\left(R+C-\frac{1}{\sqrt{2}}\right)\right) .
$$

Moreover, a quick computation shows that the radius $r$ of the biggest disc inscribed in $\Omega$ is given by

$$
r=R-\frac{1-C \sqrt{2}}{2 \sqrt{2}}
$$

Putting (4.10, 4.11) and (4.12) together yields

$$
\begin{aligned}
E(u) & =\left|(\partial \Omega)_{L}\right|+\left|(\partial \Omega)_{R}\right|-2 \pi r, \\
& =2\left(-\theta_{2} R+\sqrt{2}+(R+C) \theta_{1}-\frac{\pi C}{2}\right), \\
& =2\left(\sqrt{2}+R\left(\theta_{1}-\theta_{2}\right)+C\left(\theta_{1}-\frac{\pi}{2}\right)\right) .
\end{aligned}
$$

Now, letting $R$ tends to $+\infty$, we get the expression of the parameters in terms of the half-angle of the jump

$$
\begin{gathered}
C=\cos (X), \\
\lim _{R \rightarrow+\infty} R\left(\theta_{1}-\theta_{2}\right)=-\sin (X),
\end{gathered}
$$

and

$$
\lim _{R \rightarrow+\infty} \theta_{1}=X
$$

from which we get the expression for the energy of one cross-tie strip

$$
E(u)=2\left(\sqrt{2}-\sin (X)-\left(\frac{\pi}{2}-X\right) \cos (X)\right)
$$

which is the claim.

We have drawn in Figure 14 the pattern obtained by gluing together oblique strips constructed before and in Figures 15 and 16 reproduced several pictures of cross-tie wall (both straight and oblique) obtained on thin permalloy films and extracted from [21]. They are strikingly similar.

This paper has greatly benefited from discussions with the DKMO team (Antonio DeSimone, Bob Kohn, Stefan Müller and Felix Otto), that we wish to thank very much. We also thank R. Shäfer and Springer for their authorization to reproduce the cross-tie pictures from [21]. 


\section{REFERENCES}

[1] G. Anzelotti, S. Baldo and A. Visintin, Asymptotic behavior of the Landau-Lifschitz model of ferromagnetism. Appl. Math. Optim. 23 (1991) 171-193.

[2] L. Ambrosio, C. De Lellis and C. Mantegazza, Line energies for gradient vector fields in the plane. Calc. Var. Partial Differential Equation 9 (1999) 327-355.

[3] P. Aviles and Y. Giga, A mathematical problem related to the physical theory of liquid crystals configurations. Proc. Centre Math. Anal. Austral. Nat. Univ. 12 (1987) 1-16.

[4] P. Aviles and Y. Giga, On lower semicontinuity of a defect obtained by a singular limit of the Ginzburg-Landau type energy for gradient fields. Proc. Royal Soc. Edinburgh Sect. A 129 (1999) 1-17.

[5] L. Ambrosio, M. Lecumberry and T. Rivière, A Viscosity Property of Minimizing Micromagnetic Configurations. Preprint.

[6] N. André and I. Shafrir, On nematics stabilized by a large external field. Rev. Math. Phys. 11 (1999) 653-710.

[7] F. Bethuel, H. Brezis and F. Hélein, Ginzburg-Landau vortices. Birkhauser (1994).

[8] F. Bethuel and X. Zheng, Density of smooth functions between two manifolds in Sobolev spaces. J. Func. Anal. 80 (1988) 60-75.

[9] W. Brown, Micromagnetics. Wiley, New York (1963).

[10] G. Carbou, Regularity for critical points of a non local energy. Calc. Var. Partial Differential Equation 5 (1997) 409-433.

[11] S. Conti, I. Fonseca and G. Leoni, A $\Gamma$-convergence result for the two-gradient theory of phase transitions. Preprint.

[12] A. DeSimone, Energy minimizers for large ferromagnetic bodies. Arch. Rational Mech. Anal. 125 (1993) 99-143.

[13] B. Dacorogna and I. Fonseca, Minima absolus pour des énergies ferromagnétiques. C. R. Acad. Sci. Paris 331 (2000) $497-500$.

[14] A. DeSimone, R.V. Kohn, S. Müller and F. Otto, A compactness result in the gradient theory of phase transitions. Proc. Roy. Soc. Edinburgh 131 (2001) 833-844.

[15] A. DeSimone, R.V. Kohn, S. Müller and F. Otto, Magnetic microstructures, a paradigm of multiscale problems. Proceedings of ICIAM.

[16] A. DeSimone, R.V. Kohn, S. Müller and F. Otto, A reduced theory for thin-film micromagnetics. Preprint (2001).

[17] A. DeSimone, R.V. Kohn, S. Müller and F. Otto (in preparation).

[18] C. Evans and R. Gariepy, Measure theory and fine properties of functions. CRC Press, Boca Raton, FL, Stud. Adv. Math. (1992).

[19] R. Hardt and D. Kinderlehrer, Some regularity results in ferromagnetism. Comm. Partial Differential Equation 25 (2000) 1235-1258.

[20] F. Hang, Ph.D. Thesis. Courant Institute (2001).

[21] A. Hubert and R. Schäfer, Magnetic Domains. Springer (1998).

[22] W. Jin and R. Kohn, Singular Perturbation and the Energy of Folds. J. Nonlinear Sci. 10 (2000) 355-390.

[23] R.D. James and D. Kinderlehrer, Frustration in ferromagnetic materials, Continuum Mech. Thermodynamics 2 (1990) $215-239$.

[24] P.E. Jabin, F. Otto, and B. Perthame, Ginzburg-Landau line energies: The zero-energy case (to appear).

[25] P.E. Jabin and B. Perthame, Compactness in Ginzburg-Landau energy by kinetic averaging. Comm. Pure Appl. Math. 54 (2001) 1096-1109.

[26] M. Lecumberry and T. Rivière, Regularity for micromagnetic configurations having zero jump energy. Calc. Var. Partial Differential Equations (to appear).

[27] L. Modica and Mortola, Il limite nella $\Gamma$-convergenza di una famiglia di funzionali ellittici. Boll. Un. Mat. Ital. A (5) 14 (1977) 526-529.

[28] Y. Nakatani, Y. Uesaka and N. Hayashi, Direct solution of the Landau-Lifshitz-Gilbert equation for micromagnetics. Japanese J. Appl. Phys. 28 (1989) 2485-2507.

[29] W. Rave and A. Hubert, The Magnetic Ground State of a Thin-Film Element. IEEE Trans. Mag. 36 (2000) 3886-3899.

[30] T. Rivière and S. Serfaty, Limiting Domain Wall Energy for a Problem Related to Micromagnetics. Comm. Pure Appl. Math. 54 (2001) 294-338.

[31] T. Rivière and S. Serfaty, Compactness, kinetic formulation and entropies for a problem related to micromagnetics. Comm. in Partial Differential Equations (to appear).

[32] A. Visintin, On Landau-Lifschitz equations for ferromagnetism, Japanese J. Appl. Math. 2 (1985) 69-84.

[33] E. Sandier, preprint (1999) and habilitation thesis. University of Tours (2000).

[34] P. Sternberg, The effect of a singular perturbation on nonconvex variational problems. Arch. Rational Mech. Anal. 101 (1988) 209-260.

[35] H.A.M. Van den Berg, Self-consistent domain theory in soft micromagnetic media, II, Basic domain structures in thin film objects. J. Appl. Phys. 60 (1986) 1104-1113. 